\title{
Top-quark mass determination from $t$-channel single top production at the LHC
}

\author{
Mei Sen Gao, ${ }^{a}$ Shu Run Yuan ${ }^{a}$ and Jun Gao ${ }^{a, b, c}$ \\ ${ }^{a}$ INPAC, Shanghai Key Laboratory for Particle Physics and Cosmology, \\ School of Physics and Astronomy, Shanghai Jiao Tong University, \\ Shanghai 200240, China \\ ${ }^{b}$ Key Laboratory for Particle Astrophysics and Cosmology (MOE), \\ School of Physics and Astronomy, Shanghai Jiao Tong University, \\ Shanghai 200240, China \\ ${ }^{c}$ Center for High Energy Physics, Peking University, \\ Beijing 100871, China \\ E-mail: gmason@sjtu.edu.cn, sryuan@stu.pku.edu.cn, jung49@sjtu.edu.cn
}

ABSTRACT: We study the determination of the top-quark mass using leptonic observables in $t$-channel single top-quark production at the LHC. We demonstrate sensitivity of transverse momentum of the charged lepton on the input top-quark mass. We present predictions at next-to-next-to-leading order (NNLO) in QCD with narrow width approximation and structure function approach. Further corrections due to parton shower and hadronization, non-resonant and non-factorized contributions are discussed. To reduce impact of SM backgrounds we propose to use the charge weighted distribution for the measurement, i.e., differences between distributions of charged lepton with positive and negative charges. By modeling both signal and background processes, we found the projections for (HL-)LHC to be promising, with a total theoretical uncertainty on the extracted top-quark mass of about $1 \sim 2 \mathrm{GeV}$.

KeYwords: Heavy Quark Physics, Perturbative QCD, Quark Masses and SM Parameters ARXIV EPRINT: 2007.15527 


\section{Contents}

1 Introduction 1

2 Leptonic observables $\quad 3$

3 Theory predictions $\quad 5$

3.1 Signal regions 5

$\begin{array}{lll}3.2 & \text { NNLO predictions } & 6\end{array}$

3.3 Parametric uncertainties 9

4 Alternative theories $\quad 9$

4.1 Heavy-quark schemes 9

$\begin{array}{lll}\text { 4.2 Parton shower and hadronization } & 12\end{array}$

5 Discussions $\quad 13$

$\begin{array}{lll}5.1 & \text { Signal selection and corrections } & 14\end{array}$

$\begin{array}{lll}5.2 & \text { Non-factorized and EW corrections } & 15\end{array}$

$\begin{array}{lll}5.3 \text { Backgrounds } & 17\end{array}$

6 Projection for (HL-)LHC $\quad 19$

$\begin{array}{lll}7 & \text { Summary } & 21\end{array}$

\section{Introduction}

The top quark $(t)$ is the heaviest particle in the standard model (SM). The mass of top quark has been one of the most important input parameters of the SM and its experimental determination is crucial for precision test of the SM. For example, the recent global analysis of electroweak precision observables reveals a good agreement with top-quark mass from direct measurements [1]. Top quark also plays important role in renormalization group running of the SM especially due to the large Yukawa coupling, where the stability of the electroweak vacuum [2] is sensible to the precise top quark mass.

The mass of top-quark can be measured directly at Tevatron or LHC in top-quark pair production with subsequent decays, e.g., through invariant mass distributions of various decay products, with which the CDF, D0, ATLAS and CMS collaborations have reported an unprecedented precision of about $0.5 \mathrm{GeV}[3-5]$. The above measurements are supposed to be affected by various non-perturbative QCD effects that are modeled by MC event generators. The associated systematic uncertainties become more and more important as the experimental precision improves and have been studied extensively [6-10]. There are also discussions on intrinsic ambiguities of a precise definition of the top-quark mass due 
to infrared renormalon effects [11-13]. Many alternative methods on determination of the top-quark mass have been proposed and carried out at the LHC in order to scrutinize the experimental precision. That includes utilizing kinematic variables other than conventional invariant masses in top-quark pair production [14-19], and using measurements of total inclusive cross sections [20-22] or of distributions inclusive with respect to decay products of top quark [23-25]. There are cases of using processes of associated production of top-quark pair with a jet [26] or single top-quark production [27-29]. Besides, at future electronpositron colliders the top-quark mass can be measured much more precisely via an energy scan at threshold of top-quark pair production [30]. A recent review on various topics in determination of top-quark mass can be found in [31].

In this work we perform a theoretical study on determination of the top-quark mass through $t$-channel single top-quark production at the LHC. In particular we study in details transverse momentum distributions of the charged lepton from decay of the top quark with which we determine the top-quark mass. Similar approaches have been adopted for mass measurements in top-quark pair production [15]. It has been advertised that methods with pure leptonic variables, i.e., not directly involving jets, will be less affected by various non-perturbative effects, as well as by uncertainties from jet energy scale. Measuring top-quark mass in single top-quark production [27-29] is complementary to those measurements in pair production because of different dynamics of production including QCD color flows, which leads to different theoretical uncertainties. It can provide independent and valuable inputs to the global determination of top-quark mass. Besides, significant efforts have been made to improve the theoretical description of $t$-channel single top-quark production. We note that QCD corrections in single top-quark productions are in general much smaller than those in pair production. The next-to-leading order (NLO) QCD corrections in the 5-flavor scheme (5FS) are calculated in refs. [32-47]. The NLO calculation in the 4-flavor scheme (4FS) is carried out in ref. [48]. Full NLO corrections including topquark leptonic decay are studied within the on-shell top-quark approximation [39, 41, 43] and beyond [44, 45, 49,50]. Code for fast numerical evaluation at NLO is provided in ref. [46]. The NLO electroweak corrections are also calculated [51]. Soft gluon resummation is considered in refs. [52-58]. Matching NLO calculations to parton shower is done in the framework of POWHEG and MC@NLO [49, 59-61]. Next-to-next-to-leading order (NNLO) QCD corrections with a stable top quark are calculated in ref. [62]. The study here are based on the NNLO calculations including top-quark leptonic decay under narrow width approximation (NWA) as developed in refs. [63-66] that provide a realistic partonlevel simulation at NNLO. Moreover, we have used the structure function approach [67-69], where the $t$-channel production can be factorized into two charged-current deep-inelastic scatterings with light quarks and heavy quarks respectively. Gluon exchanges between the two quark lines contribute at NNLO. They are suppressed by QCD colors and neglected in our study. Progresses on calculation of those corrections have been made in [70, 71].

However, we should point out several potential problems on determination of top-quark mass via single top-quark production. It suffers from large backgrounds due to top-quark pair production as well as production of $\mathrm{W} / \mathrm{Z}$ boson with jets. A pure signal sample can only be obtained in a signal-enriched fiducial volume as shown in the ATLAS and CMS 
measurements $[27,28]$. That indicates the measurements are less inclusive and also have relatively low statistics as compared to measurements in pair production. The former one is less concerned as far as precise theory predictions can be provided which is the main topic of this paper. The shortcoming on statistics can also be overcome thanks to the high luminosity of LHC.

The rest of our paper is organized as follows. In section 2, we describe leptonic observables and the sensitivity to top-quark mass. In section 3, we present our nominal predictions on leptonic distributions including their intrinsic and parametric uncertainties. Section 4 provides results of alternative theory predictions including those from different heavy quark schemes and from MC generators. In section 5 we address further questions on both theory and experimental sides related to the measurement and in section 6 we show our projected precision of measurements at (HL-)LHC. Finally our summary and conclusions are presented in section 7 .

\section{Leptonic observables}

We demonstrate dependence of leptonic variables on the top quark mass in single top-quark production. We use on-shell renormalization scheme in perturbative calculations. Thus the top-quark mass we refer to in the remaining sections is always the pole mass. Specifically we focus on transverse momentum distributions of the charged lepton.

We start with a pedagogical discussion based on a calculation at Born level. Kinematic distributions of the charged-lepton can be understood as below. For decay of an on-shell top quark in its rest frame, the triple differential decay width can be expressed as [72]

$$
\frac{d \Gamma}{d x d y d \cos \theta}=\frac{G_{F}^{2} m_{t}^{5}}{32 \pi^{3}} \frac{\left|V_{t b}\right|^{2}}{(1-y / \bar{y})^{2}+\gamma^{2}} x\left(x_{m}-x\right)(1+S \cos \theta),
$$

with

$$
x_{m}=1-\epsilon^{2}, \epsilon=m_{b} / m_{t}, \bar{y}=m_{W}^{2} / m_{t}^{2}, \gamma=\Gamma_{W} / m_{W},
$$

where $m_{b}, m_{t}, m_{W}$ and $\Gamma_{W}$ are masses of the bottom quark, top quark and $W$ boson, and the width of the $W$ boson, respectively. $G_{F}$ and $V_{t b}$ are the Fermi constant and the Cabibbo-Kobayashi-Maskawa (CKM) matrix element. The three kinematic variables are $x=2 E_{l} / m_{t}, y=M_{l \nu}^{2} / m_{t}^{2}$, and the cosine between directions of the charged lepton and the spin axis of the top quark. $S=1(0)$ corresponds to top quark being fully (un)polarized. The Dalitz variables $x$ and $y$ fulfill kinematic constraints

$$
0 \leq y \leq(1-\epsilon)^{2}, \omega_{-} \leq x \leq \omega_{+}
$$

with $\omega_{-}=1-p_{0}-p_{3}, \omega_{+}=1-p_{0}+p_{3}$, and $p_{0}=\left(1-y+\epsilon^{2}\right) / 2, p_{3}=\sqrt{p_{0}^{2}-\epsilon^{2}}$. Distribution of transverse momentum of the charged lepton $\left(p_{T, l}\right)$ defined as with respect to axis $z$ can be derived from eq. (2.1). For simplicity we first assume a zero width of the $W$ boson and a massless bottom quark. For unpolarized top quark average transverse momentum of the charged lepton can be calculated as

$$
\left\langle p_{T, l}\right\rangle \equiv \frac{\int p_{T, l} d \Gamma}{\int d \Gamma}=\frac{\pi}{16} \frac{1+2 \bar{y}+3 \bar{y}^{2}}{1+2 \bar{y}} m_{t} .
$$


It is straightforward to show that eq. (2.4) also holds for a polarized top quark with the spin axis not necessarily coincident with the $z$ axis. Substituting mass values of $m_{t}=172.5 \mathrm{GeV}$ and $m_{W}=80.385 \mathrm{GeV}$ [73], we arrive at

$$
\left\langle p_{T, l}\right\rangle=37.21\left(1+0.695 \frac{\delta m_{t}}{172.5 \mathrm{GeV}}\right) \mathrm{GeV},
$$

assuming a small shift of the top-quark mass of $\delta m_{t}$. Thus a $1 \mathrm{GeV}$ shift of the top-quark mass translates into a $0.4 \%$ change of the average transverse momentum. Effects of the finite $W$ boson width can be included by integrating fully in $y$ instead of using narrow width approximation. The average transverse momentum is increased by one permille for a $W$ boson width of $2.2 \mathrm{GeV}$ as compared to NWA,

$$
\left\langle p_{T, l}\right\rangle=37.21\left(1+0.0009 \frac{\Gamma_{W}}{2.2 \mathrm{GeV}}\right) \mathrm{GeV} .
$$

Effects due to the finite bottom quark mass are expected to be even smaller with the result given by

$$
\left\langle p_{T, l}\right\rangle=37.21\left(1-0.0004 \frac{m_{b}}{4.5 \mathrm{GeV}}\right) \mathrm{GeV} .
$$

At hadron colliders kinematics of the charged lepton can be measured in both the single top-quark production and top-quark pair production in which the top quarks are boosted in general. For a boost along the $z$ axis it will not affect the transverse momentum distributions of the decay products. Now considering the top quark travels perpendicularly to the $z$ axis with a velocity $\beta$, the average $p_{T, l}$ from the decay of an unpolarized top quark is given by

$$
\left\langle p_{T, l}\right\rangle=37.21 \frac{\left(1-0.0015 \beta+0.257 \beta^{2}\right)}{\sqrt{1-\beta^{2}}} \mathrm{GeV}
$$

as derived from eq. (2.1) keeping up to $\mathcal{O}\left(\beta^{2}\right)$ terms in the numerator. At the LHC $13 \mathrm{TeV}$ the top quark in $t$-channel singly production has an average $p_{T}$ of about $40 \mathrm{GeV}$, while the average is about $120 \mathrm{GeV}$ in pair production. They correspond to roughly a velocity of top quark of 0.2 and 0.6 respectively. From direct calculations of production with subsequent leptonic decay of the top quark at leading order (LO), we obtain the following results for LHC $13 \mathrm{TeV}$,

$$
\left\langle p_{T, l}\right\rangle_{t-c h}=38.38 \mathrm{GeV},\left\langle p_{T, l}\right\rangle_{t \bar{t}}=56.37 \mathrm{GeV},
$$

which are in agreement with estimations using eq. (2.8) together with the corresponding velocities at the LHC.

From above discussion we understood the precise distributions of the charged lepton will depend on modeling of the top-quark kinematics and polarizations in the production. They can be sensitive to QCD corrections in production and in decay of the top quark. Besides, in experimental measurements various selection cuts are applied that can further change distributions of the charged lepton. 


\section{Theory predictions}

In this section we present our main results on predictions of the leptonic observables. We first identify the signal regions used for the LHC measurement and show the sensitivity of the proposed observable to the top-quark mass. Then we present our theory predictions based on a next-to-next-to-leading order calculation including decay of the top quark. Discussions on scale variations and parametric uncertainties are also included.

\subsection{Signal regions}

In experimental measurements various kinematic cuts are applied due to finite coverage of detectors, such as transverse momentums or rapidities of the reconstructed jets, electrons, muons and photons. For the single top-quark production, additional cuts or selections are required in order to suppress SM backgrounds from the top-quark pair production and associated production of $W / Z$ bosons and jets. We follow closely fiducial regions used in the CMS analyses at 8 and $13 \mathrm{TeV}[28,74]$.

We require one charged lepton in the final state with $p_{T}>26 \mathrm{GeV}$ and $|\eta|<2.4$, and include only one family of leptons from decay of the top quark in results through the paper unless otherwise specified. We use the anti- $k_{T}$ jet algorithm [75] with a distance parameter of $\mathrm{D}=0.4$. Jets are required to have $p_{T}>40 \mathrm{GeV}$ and $|\eta|<4.7$. A clustered jet at parton level is defined as $b$-tagged if it has a non-zero net bottom-quark number in the constituents and further has $|\eta|<2.4$. In addition a constant $b$-tagging efficiency of $50 \%$ has been applied. Light jets are defined as jets that are not $b$-tagged. We consider two signal regions for $t$-channel production, CMS-SA and CMS-SB. Both are required to have exactly two jets in the final state with one being a $b$-tagged jet and the other being a light jet. We require the transverse mass of the charged lepton and the missing transverse momentum from neutrinos to be greater than $50 \mathrm{GeV}$. In CMS-SB the light jet is required to stay in the forward region, namely $|y|>2.5$, which can further increase the signal to background ratio.

We demonstrate the sensitivity of the leptonic distributions to the top-quark mass in figure 1 for LHC $13 \mathrm{TeV}$. We show transverse momentum distributions of the charged lepton in the two signal regions with a top-quark mass of $172.5 \mathrm{GeV}$ or shifted by $5 \mathrm{GeV}$, calculated at NLO in QCD. The lower inset shows ratios of the distributions with different top-quark masses. Details of the calculation will be explained later. The increase of topquark mass leads to a harder $p_{T}$ spectrum in general. At very large $p_{T}$, enhancements of the distribution are cancelled out because of the increasing importance of top-quark kinematics from production. Results of two signal regions show a very similar dependence on the top-quark mass.

We prefer to use a single variable to extract the top-quark mass, rather than from a template fit to the full leptonic distribution. We choose the variable as average $p_{T}$ of the charged lepton. We can select different windows of the $p_{T}$ spectrum to be included. We plot relative change of the average $p_{T}$ when varying the top-quark mass by $1 \mathrm{GeV}$, as a function of an upper limit placed on $p_{T}$ in figure 2. For both signal regions the sensitivity saturates to a value of about $0.3 \%$ when the upper limit reaches above $100 \mathrm{GeV}$. In latter sections we 

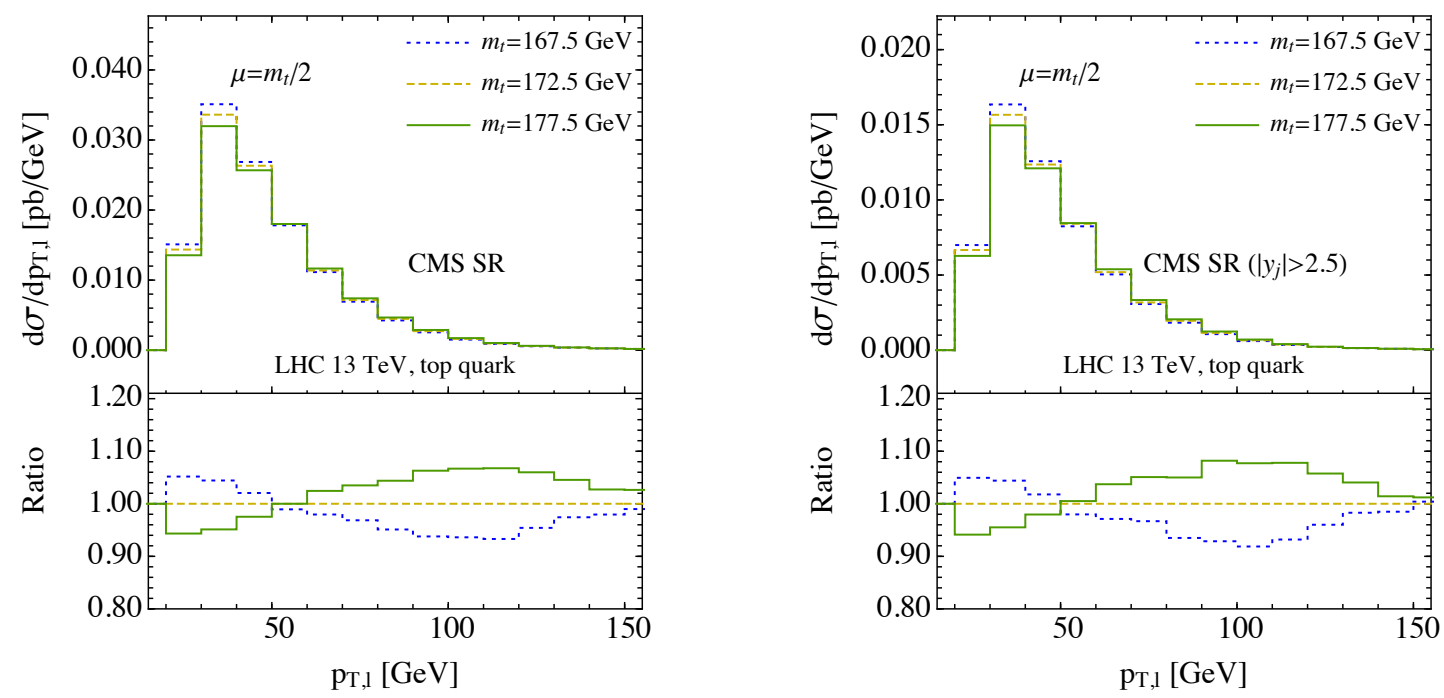

Figure 1. Transverse momentum distribution of the charged lepton within the two fiducial regions at NLO in QCD for different choices of top-quark mass, calculated in the 5FS for LHC $13 \mathrm{TeV}$.

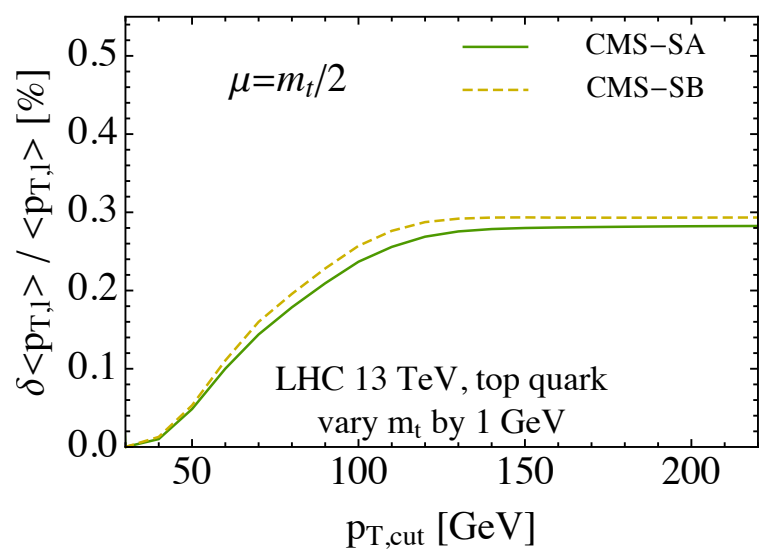

Figure 2. Induced change of the average transverse momentum of the charged lepton within the two fiducial regions when varying the top-quark mass by $1 \mathrm{GeV}$, as a function of an upper limit on the transverse momentum, calculated at NLO in QCD in the 5FS for LHC $13 \mathrm{TeV}$.

will present results for two representative values of the upper limit, $100 \mathrm{GeV}$ and $200 \mathrm{GeV}$, respectively. Inclusion of high $p_{T}$ region usually leads to larger theory uncertainties.

\section{$3.2 \quad$ NNLO predictions}

NNLO predictions for the $t$-channel single top-quark production in the 5 -flavor scheme are calculated using the phase-space slicing with the $N$-jettiness variable [76-79] together with the method of "projection-to-Born" in ref. [80]. Details for the NNLO calculation in the 5FS can be found in refs. $[63,64]$. In the calculation, QCD corrections can be factored as from ei- 


\begin{tabular}{|l|cc|cc|}
\hline \multirow{2}{*}{$\begin{array}{l}\left\langle p_{T, l}\right\rangle \\
{[\mathrm{GeV}]}\end{array}$} & \multicolumn{2}{|c}{ CMS-SA } & \multicolumn{2}{c|}{ CMS-SB } \\
\cline { 2 - 5 } & $<100 \mathrm{GeV}$ & $<200 \mathrm{GeV}$ & $<100 \mathrm{GeV}$ & $<200 \mathrm{GeV}$ \\
\hline $\mathrm{LO}$ & $47.33_{-0.03}^{+0.03}(47.33)$ & $49.31_{-0.08}^{+0.09}(49.31)$ & $47.38_{-0.02}^{+0.01}(47.38)$ & $48.73_{-0.04}^{+0.05}(48.73)$ \\
\hline $\mathrm{NLO}$ & $47.78_{-0.14}^{+0.17}(48.06)$ & $50.37_{-0.30}^{+0.38}(50.67)$ & $47.49_{-0.09}^{+0.13}(47.84)$ & $49.66_{-0.27}^{+0.36}(50.02)$ \\
\hline NNLO & $47.65_{-0.03}^{+0.09}(48.01)$ & $50.10_{-0.16}^{+0.09}(50.49)$ & $47.35_{-0.03}^{+0.14}(47.75)$ & $49.25_{-0.12}^{+0.17}(49.67)$ \\
\hline
\end{tabular}

Table 1. Average transverse momentum of the charged lepton within the two fiducial regions at various orders in QCD in the $5 \mathrm{FS}$ for $\mathrm{LHC} 13 \mathrm{TeV}$. Scale variations are evaluated by taking envelop of results with 9 scale choices. Numbers in parenthesis correspond to predictions without including QCD corrections in decay of top quark.

ther fermion line with heavy quarks or light quarks neglecting certain color suppressed contributions [67-69]. We also include consistently the NNLO corrections in decay of the top quark as originally calculated in [81] using narrow width approximation. We focus on predictions for the top-quark production at LHC $13 \mathrm{TeV}$. Results for top anti-quark production can be obtained through a CP transformation with substitutions of the parton distributions.

We use a PDF set of PDF4LHC15_nnlo_30 with $\alpha_{S}\left(m_{Z}\right)=0.118$ [82-87], and a nominal value of the top-quark mass of $172.5 \mathrm{GeV}$. The central scales of QCD renormalization and factorization are set to half of the top-quark mass. A lower value of the QCD scale in 5FS was suggested in ref. [88] which shows those quasi-collinear logarithms to be resummed are accompanied by a universal suppression from phase space integration, as also supported by numerical calculations in [66]. We evaluate scale uncertainty by varying the two scales independently with a factor of two and taking the envelope of results with 9 scale choices. Effects due to finite width of the $W$ boson, finite mass of the bottom quark in top-quark decay, and finite width of the top quark, are included by adding their corrections calculated at leading order. For example, the off-shell effects of top quark are modeled with a Breit-Wigner shape at LO. The resulted average $p_{T}$ of the charged lepton differs with that in the NWA by $0.03 \mathrm{GeV}$, which are added into our final NNLO predictions calculated with NWA. We will discuss off-shell effects beyond leading order in section 5.2.

We show transverse momentum distributions of the charged lepton in the two signal regions in figure 3 at various orders in QCD together with scale uncertainties for LHC $13 \mathrm{TeV}$. In the lower inset of each plot we show ratios of the predictions to a common reference calculated at NNLO with nominal scale choice. The NNLO corrections lead to a softer spectrum due to both soften of the top-quark $p_{T}$ and additional radiations in top-quark decay [81]. Size of the NNLO corrections ranges from $-5 \%$ to $-35 \%$ for the $p_{T}$ region shown. Moderate reduction of scale uncertainties are seen when including the NNLO corrections. However, the scale variations at NLO underestimate the size of NNLO corrections for the signal region CMS-SB especially in the high- $p_{T}$ tail.

We present detailed results on the average $p_{T}$ of the charged lepton in table 1 for the two signal regions and with two choices of the upper limit on $p_{T}$. Numbers in parenthesis correspond to predictions when excluding QCD corrections in decay of the top quark, i.e., only including corrections in production of the top quark. We find the leading order 

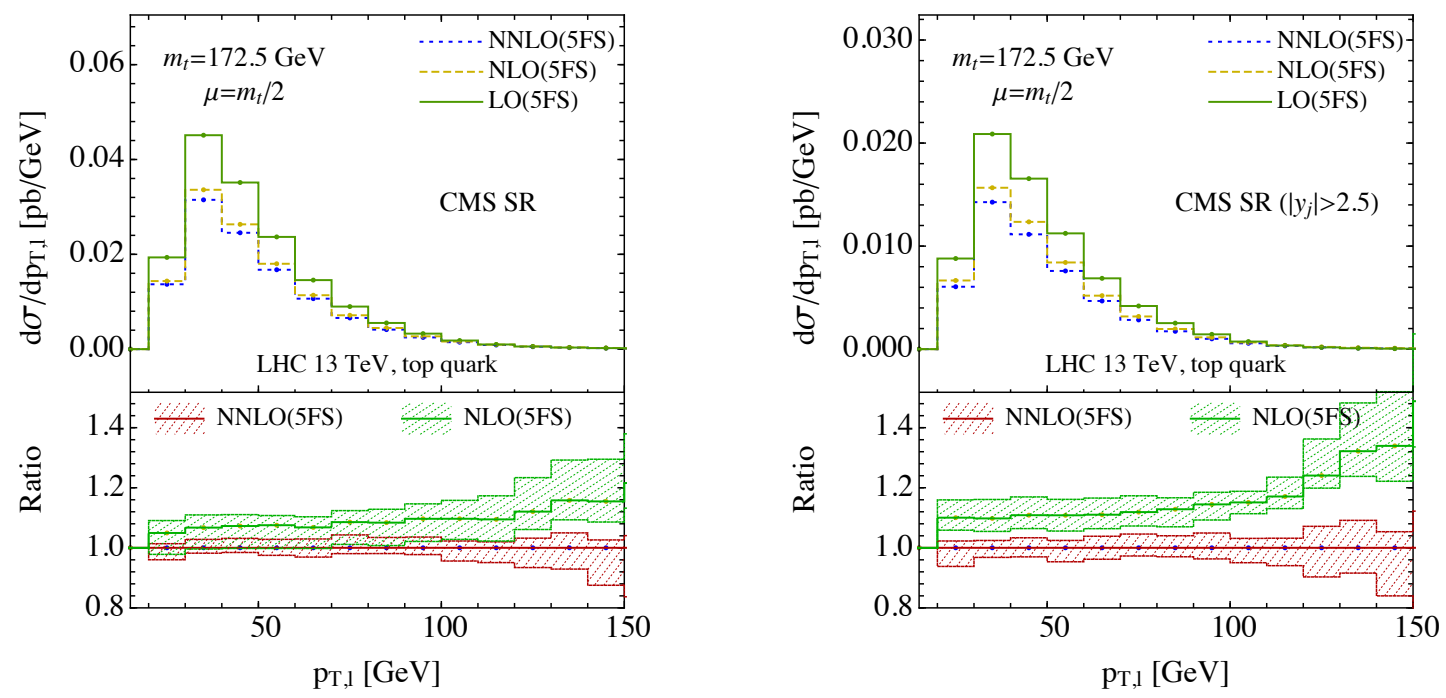

Figure 3. Transverse momentum distribution of the charged lepton within the two fiducial regions at various orders in QCD in the $5 \mathrm{FS}$ for $\mathrm{LHC} 13 \mathrm{TeV}$. Scale variations are evaluated by taking envelop of results with 9 scale choices.

predictions show a rather small scale variation, which can be understood since the change of scales at LO only impact the overall normalization and longitudinal boost of the system, not the shape of the transverse momentum distribution. The LO predictions can not describe well distributions of transverse momentum of the top quark, especially at high $p_{T}$, as explained in [66]. The NNLO corrections lead to a reduction of the average $p_{T}$ by less than $0.2 \mathrm{GeV}$ if the upper limit of $100 \mathrm{GeV}$ is applied. The corrections are slightly larger if instead the upper limit of $200 \mathrm{GeV}$ is used. The final NNLO predictions show scale uncertainties at the level of $0.1 \mathrm{GeV}$ which are comparable to the change as induced by a shift of the top-quark mass of $1 \mathrm{GeV}$.

QCD corrections from top-quark decay are large comparing to our target precision of the average $p_{T}$. They reduce the average $p_{T}$ by about $0.3 \sim 0.4 \mathrm{GeV}$ at NLO. The NNLO corrections from top-quark decay further decrease the average $p_{T}$ by $0.1 \mathrm{GeV}$ in the case of signal region CMS-SA. We recall that NNLO corrections due to top-quark decay consist of two parts, one from pure two-loop corrections in top-quark decay and the other from one-loop corrections in decay combined with one-loop corrections in production. Both of the two pieces are important. Cancellation between them may occur depending on the observables and kinematic region considered. We also show predictions on total fiducial cross sections in table 2. The NNLO corrections reduce the cross sections by about $6 \%$ for signal region CMS-SA, with predictions located at the lower boundary of the NLO scale variations. The reduction is about $10 \%$ for the signal region CMS-SB. 


\begin{tabular}{|c|c|c|c|c|}
\hline \multirow{2}{*}{$\begin{array}{l}\sigma_{f i d} \\
{[\mathrm{pb}]}\end{array}$} & \multicolumn{2}{|c|}{ CMS-SA } & \multicolumn{2}{|c|}{ CMS-SB } \\
\hline & $<100 \mathrm{GeV}$ & $<200 \mathrm{GeV}$ & $<100 \mathrm{GeV}$ & $<200 \mathrm{GeV}$ \\
\hline $\mathrm{LO}$ & $1.55_{-0.21}^{+0.16}(1.55)$ & $1.59_{-0.22}^{+0.17}(1.59)$ & $0.724_{-0.08}^{+0.06}(0.724)$ & $0.739_{-0.08}^{+0.06}(0.739)$ \\
\hline $\mathrm{NLO}$ & $1.17_{-0.08}^{+0.03}(1.31)$ & $1.22_{-0.08}^{+0.03}(1.36)$ & $0.545_{-0.02}^{+0.02}(0.613)$ & $0.562_{-0.02}^{+0.02}(0.632)$ \\
\hline NNLO & $1.10_{-0.02}^{+0.02}(1.24)$ & $1.14_{-0.02}^{+0.02}(1.29)$ & $0.493_{-0.01}^{+0.01}(0.563)$ & $0.506_{-0.01}^{+0.01}(0.579)$ \\
\hline
\end{tabular}

Table 2. Fiducial cross section at various orders in QCD in the $5 F$ S for LHC $13 \mathrm{TeV}$. Scale variations are evaluated by taking the envelop of results with 9 scale choices. Numbers in parenthesis correspond to predictions without including QCD corrections in decay of top quark.

\subsection{Parametric uncertainties}

We investigate dependence of our predictions on various inputs and the associated parametric uncertainties. That includes the parton distribution functions, QCD coupling constant, and bottom quark mass. Uncertainties due to parton distribution functions are estimated following the PDF4LHC recommendation [82] and using PDF4LHC15_nnlo_30 PDF set. We calculate the dependence on QCD coupling constant by varying $\alpha_{S}\left(m_{Z}\right)$ by \pm 0.0015 from its nominal value of 0.118 , and using PDFs of the same $\alpha_{S}\left(m_{Z}\right)$ values. We use MMHT2014 PDF set [89] with different bottom-quark masses to calculate the changes when varying the pole mass of bottom quark by $0.5 \mathrm{GeV}$. The impact on average $p_{T}$ of the charged lepton and on the total fiducial cross section are summarized in table 3 . We also include corresponding numbers when varying the mass of top quark by $1 \mathrm{GeV}$ for comparison.

We find in all cases the parametric uncertainties on average $p_{T}$ are at the level of $0.01 \sim 0.02 \mathrm{GeV}$, and are small comparing to the dependence on the top-quark mass. On another hand, the total fiducial cross sections show larger uncertainties. For example, the PDF uncertainties are $2 \% \sim 4 \%$, and the uncertainties due to bottom quark mass are $1 \% \sim 3 \%$ if taking error of bottom quark mass as $0.2 \mathrm{GeV}$ [89]. The total fiducial cross sections are insensitive to the top-quark mass, unlike the average $p_{T}$ of the charged lepton.

\section{Alternative theories}

We present two alternative theory predictions concerning both the perturbative and nonperturbative components in $t$-channel production of single top quark. Comparison with our nominal predictions can lead a better understanding on the related theoretical uncertainties.

\subsection{Heavy-quark schemes}

It is known that the $t$-channel production can also be calculated in a factorization scheme with a fixed 4 light-quark flavors. The 5FS has the advantages of resumming large logarithms of bottom-quark mass due to gluon splitting into bottom quarks from the initial state. The 4FS maintains full bottom quark mass dependence through fixed order with current predictions available only at NLO in QCD. We note that leading order calculations in $4 \mathrm{FS}$ already contain ingredients appearing at next-to-leading order in 5FS. 


\begin{tabular}{|l|c|cc|cc|}
\hline \multirow{2}{*}[\mathrm{GeV}]{$/[\mathrm{pb}]$} & \multicolumn{2}{|c|}{ CMS-SA } & \multicolumn{2}{c|}{ CMS-SB } \\
\cline { 3 - 6 } & & $<100 \mathrm{GeV}$ & $<200 \mathrm{GeV}$ & $<100 \mathrm{GeV}$ & $<200 \mathrm{GeV}$ \\
\hline \multirow{2}{*}{ PDFs(68\% C.L.) $)$} & $\delta\left\langle p_{T, l}\right\rangle$ & 0.014 & 0.023 & 0.021 & 0.022 \\
& $\delta \sigma_{f i d}$ & 0.040 & 0.041 & 0.020 & 0.021 \\
\hline \multirow{2}{*}{$\alpha_{S}\left(m_{Z}\right)(0.0015)$} & $\delta\left\langle p_{T, l}\right\rangle$ & $<0.01$ & $<0.01$ & $<0.01$ & $<0.01$ \\
& $\delta \sigma_{f i d}$ & 0.017 & 0.018 & 0.005 & 0.005 \\
\hline \multirow{2}{*}{$m_{b}(0.5 \mathrm{GeV})$} & $\delta\left\langle p_{T, l}\right\rangle$ & $<0.01$ & $<0.01$ & $<0.01$ & $<0.01$ \\
& $\delta \sigma_{f i d}$ & 0.064 & 0.066 & 0.029 & 0.030 \\
\hline \multirow{2}{*}{$m_{t}(1.0 \mathrm{GeV})$} & $\delta\left\langle p_{T, l}\right\rangle$ & 0.11 & 0.14 & 0.12 & 0.14 \\
& $\delta \sigma_{f i d}$ & 0.0039 & 0.0035 & 0.0013 & 0.0011 \\
\hline
\end{tabular}

Table 3. PDF uncertainties on the average transverse momentum of the charged lepton and on the fiducial cross section within the two fiducial regions, followed by induced changes on the same quantities when varying $\alpha_{S}\left(m_{Z}\right), m_{b}$, and $m_{t}$ by the amount in parenthesis.

Critical questions arise on the use and agreement of the two heavy-quark schemes in $t$ channel single top-quark production, with efforts at understanding made in refs. [48, 88, 90]. In a recent study by one of the authors [66], we compare predictions at NNLO in 5FS to those at NLO in 4FS without decaying of the top quark. We found the two schemes agree within a few percent in general for the shape of kinematic distributions of the top quark, and differ on the overall normalizations. We conclude that 5FS provides a better modeling on $t$-channel production when both are evaluated at comparable perturbative orders. Here we extend the comparison to include leptonic decay of the top quark, focusing on the leptonic observables discussed.

We use MCFM [91, 92] program to calculate $t$-channel single top-quark production with subsequent decays in the 4FS. The original calculation was detailed in ref. [48]. We use CT14 NNLO PDFs [86] with 4 light-quark flavors through the comparison and a bottomquark mass of $4.75 \mathrm{GeV}$. We set the nominal QCD renormalization scale and factorization scale to half of the top-quark mass. Scale variations are evaluated with the 9 -scales envelope same as before.

We show 4FS predictions on transverse momentum distributions of the charged lepton in figure 4 for the two fiducial regions, compared with the NNLO predictions in 5FS. In the comparison the NLO predictions in 4FS include the NLO corrections in top-quark decay, and the NNLO predictions in 5FS include further NNLO corrections in decay. We find the LO and NLO predictions in $4 \mathrm{FS}$ show less differences as compared to the case of stable top quark in ref. [66]. That is because of the jet veto condition applied, namely requiring exactly two jets in the final state. For the same reason the NLO predictions in 4FS agree well with NNLO ones in 5FS even for the overall normalizations. Shape differences of the two predictions can be understood as due to both the harder $p_{T}$ spectrum of the top quark from production in $4 \mathrm{FS}$ and the inclusion of NNLO corrections from decay in 5FS. The scale variations are slightly larger in the high $p_{T}$ region for the $4 \mathrm{FS}$ predictions. 

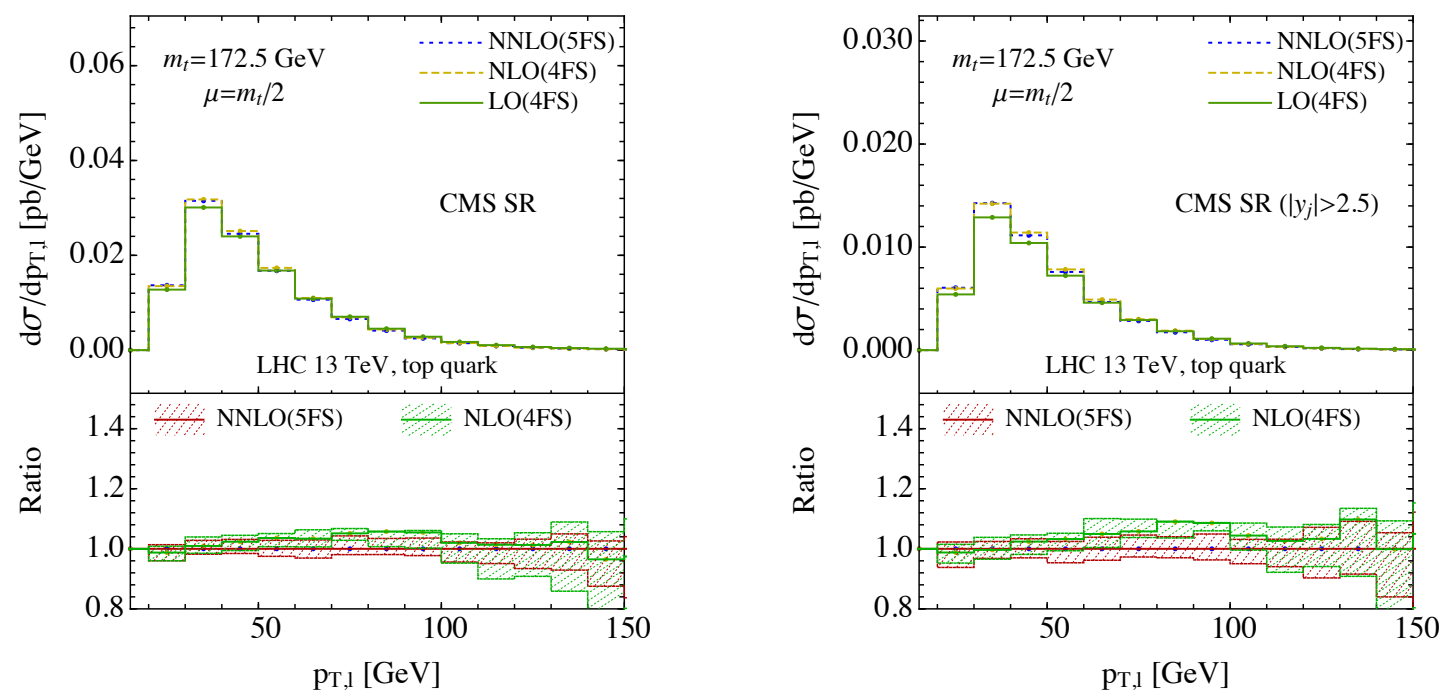

Figure 4. Transverse momentum distribution of the charged lepton within the two fiducial regions at various orders in QCD for LHC $13 \mathrm{TeV}$, comparing $5 \mathrm{FS}$ with $4 \mathrm{FS}$. Scale variations are evaluated by taking the envelop of results with 9 scale choices.

\begin{tabular}{|l|cc|cc|}
\hline \multirow{2}{*}{$\begin{array}{l}\left\langle p_{T, l}\right\rangle \\
{[\mathrm{GeV}]}\end{array}$} & \multicolumn{2}{|c}{ CMS-SA } & \multicolumn{2}{c|}{ CMS-SB } \\
\cline { 2 - 5 } & $<100 \mathrm{GeV}$ & $<200 \mathrm{GeV}$ & $<100 \mathrm{GeV}$ & $<200 \mathrm{GeV}$ \\
\hline $\mathrm{LO}(4 \mathrm{FS})$ & $48.45_{-0.05}^{+0.02}(48.45)$ & $51.65_{-0.14}^{+0.12}(51.65)$ & $48.22_{-0.06}^{+0}(48.22)$ & $50.79_{-0.10}^{+0}(50.79)$ \\
\hline $\mathrm{NLO}(4 \mathrm{FS})$ & $47.94_{-0.18}^{+0.08}(48.11)$ & $50.33_{-0.49}^{+0.23}(50.55)$ & $47.79_{-0.20}^{+0.07}(47.89)$ & $49.72_{-0.44}^{+0.20}(49.83)$ \\
\hline $\mathrm{NNLO}(5 \mathrm{FS})$ & $47.65_{-0.03}^{+0.09}(48.01)$ & $50.10_{-0.16}^{+0.09}(50.49)$ & $47.35_{-0.03}^{+0.14}(47.75)$ & $49.25_{-0.12}^{+0.17}(49.67)$ \\
\hline
\end{tabular}

Table 4. Average transverse momentum of the charged lepton within the two fiducial regions at various orders in QCD for LHC $13 \mathrm{TeV}$, comparing $5 \mathrm{FS}$ with 4FS. Scale variations are evaluated by taking the envelop of results with 9 scale choices. Numbers in parenthesis correspond to predictions without including QCD corrections in decay of top quark.

More comparisons can be found in table 4 for the average $p_{T}$ of the charged lepton. The numbers in parenthesis correspond to predictions without including QCD corrections in decay of top quark. We find scale variations of LO predictions in 4FS are small and underestimate the genuine NLO corrections especially when including high- $p_{T}$ regions, similar to the case of LO predictions in $5 \mathrm{FS}$. The difference on average $p_{T}$ between NLO predictions in $4 \mathrm{FS}$ and NNLO predictions in $5 \mathrm{FS}$ is about $0.3 \mathrm{GeV}$ for the fiducial region CMS-SA and $0.4 \mathrm{GeV}$ for the fiducial region CMS-SB. Half of the difference can be attributed to the different treatment on corrections in decay of the top quark. Scale variations are slightly larger for average $p_{T}$ from NLO predictions in $4 \mathrm{FS}$. Predictions of the two schemes overlap in general once considering both scale variations. Similar results for the total fiducial cross sections are shown in table 5 where even better agreement are seen between the two schemes. 


\begin{tabular}{|c|c|c|c|c|}
\hline \multirow{2}{*}{$\begin{array}{l}\sigma_{f i d} \\
{[\mathrm{pb}]}\end{array}$} & \multicolumn{2}{|c|}{ CMS-SA } & \multicolumn{2}{|c|}{ CMS-SB } \\
\hline & $<100 \mathrm{GeV}$ & $<200 \mathrm{GeV}$ & $<100 \mathrm{GeV}$ & $<200 \mathrm{GeV}$ \\
\hline $\mathrm{LO}(4 \mathrm{FS})$ & $1.08_{-0.12}^{+0.16}(1.08)$ & $1.13_{-0.13}^{+0.16}(1.13)$ & $0.464_{-0.06}^{+0.07}(0.464)$ & $0.480_{-0.06}^{+0.08}(0.480)$ \\
\hline $\mathrm{NLO}(4 \mathrm{FS})$ & $1.12_{-0.02}^{+0.02}(1.18)$ & $1.16_{-0.02}^{+0.02}(1.22)$ & $0.503_{-0.01}^{+0.01}(0.528)$ & $0.517_{-0.01}^{+0.01}(0.543)$ \\
\hline NNLO $(5 \mathrm{FS})$ & $1.10_{-0.02}^{+0.02}(1.24)$ & $1.14_{-0.02}^{+0.02}(1.29)$ & $0.493_{-0.01}^{+0.01}(0.563)$ & $0.506_{-0.01}^{+0.01}(0.579)$ \\
\hline
\end{tabular}

Table 5. Fiducial cross section at various orders in QCD for LHC $13 \mathrm{TeV}$, comparing $4 \mathrm{FS}$ with 5 FS. Scale variations are evaluated by taking the envelop of results with 9 scale choices. Numbers in parenthesis correspond to predictions without including QCD corrections in decay of top quark.

\subsection{Parton shower and hadronization}

We compare our parton-level results with those from various Monte Carlo generators both in 5-flavor number scheme. We calculate the fiducial cross sections and distributions at NLO in QCD matched with parton shower using MG5_aMC@NLO program [93]. We generate matched events with stable top quarks that are further decayed with MadSpin [94]. Events are then passed to various generators for parton shower and hadronization, including PYTHIA6 [95], PYTHIA8 [96], and HERWIG7 [97]. Finally the events are analysed with MadAnalysis5 [98] and FastJet [99]. We use same input parameters as in previous fixedorder calculations for PDFs, QCD scales and selection cuts. We have checked the total inclusive cross sections agree at NLO in the two calculations. In MC simulations one difference with respect to fixed-order calculation is on definition of $b$-tagged jet for which we use the default method implemented in MadAnalysis5. Be specific, for each event after jet clustering, one searches for intermediate $b$ quarks in the MC record. The clustered jets are considered as a $b$-tagged jet if it can be associated with a MC $b$ quark inside the jet cone. Similarly a $b$-tagging efficiency of $50 \%$ is applied on the $b$-tagged jet.

We show MC predictions on transverse momentum distributions of the charged lepton in figure 5 for the two fiducial regions, comparing with the NNLO predictions calculated earlier. We find HERWIG7 provides different predictions comparing with PYTHIA6 and PYTHIA8 while the latter two show very good agreement. That can be due to different shower algorithms or possibly the way of different shower programs handling decayed resonance, for example as studied in refs. [49, 100]. We can also compare MC predictions with the NNLO predictions. It is interesting that the two show very good agreement on shape of the distribution though the normalization is higher by about $10 \%$ for PYTHIA6 and PYTHIA8. Such agreement is non-trivial since the matrix elements used in MC predictions do not include NLO corrections in top-quark decay which have large impact on the $p_{T}$ distribution as shown in table 1. Parton shower resummation takes into account part of the missing NLO and NNLO corrections and brings the MC predictions closer to the NNLO fixed-order predictions.

Comparison on the average $p_{T}$ of the charged lepton are summarized in table 6 along with the fiducial cross section shown in table 7 . In both tables we also include NLO fixedorder predictions with and without corrections in decay of the top quark. As a cross check 

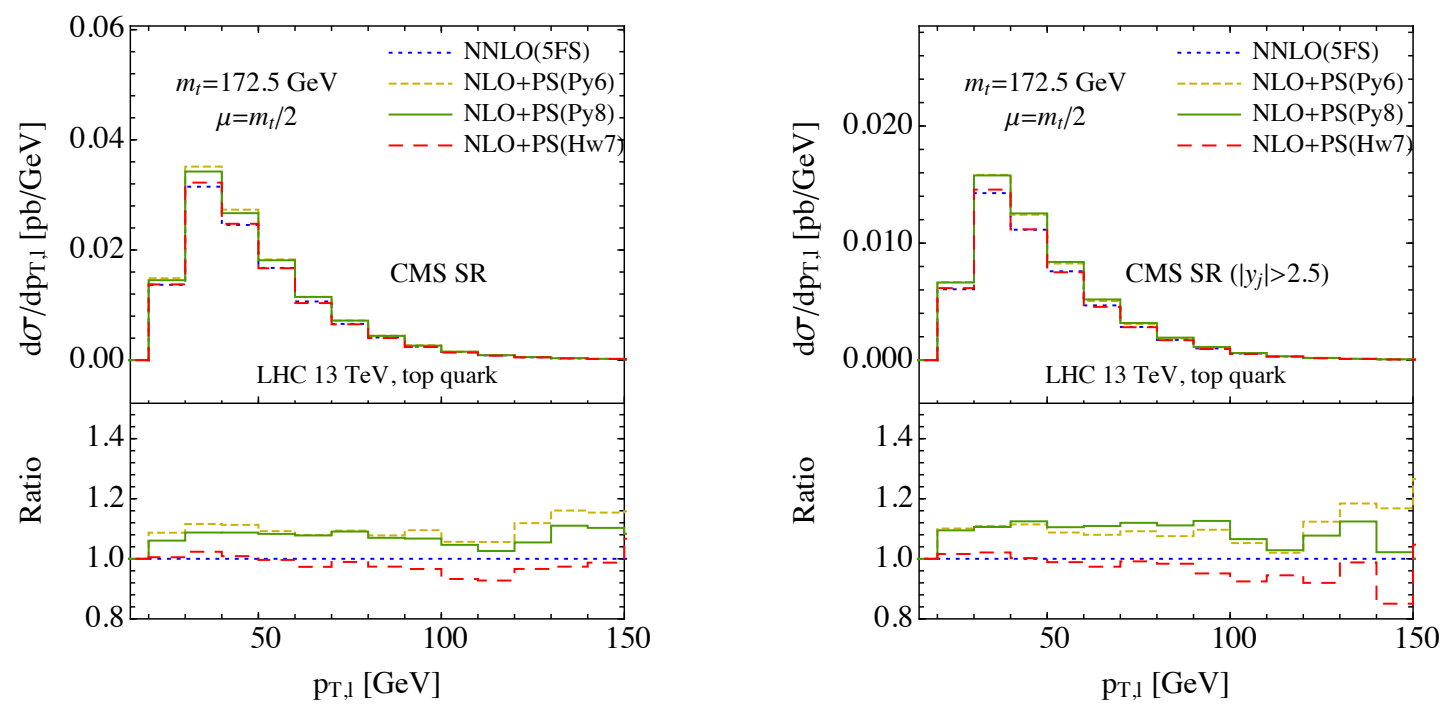

Figure 5. Transverse momentum distribution of the charged lepton within the two fiducial regions, comparing predictions at fixed-order and those from various event generators in the 5FS for LHC $13 \mathrm{TeV}$.

we show the NLO predictions calculated in 5FS with MCFM and NWA, which agree well with our results. The small differences are due to off-shell effects at LO included in our results as mentioned earlier. For MC predictions, numbers in parenthesis correspond to turning hadronizaiton off in the generators. The hadronization corrections are small for all generators considered, within the statistical uncertainties which are about $0.03 \mathrm{GeV}$ for the average $p_{T}$. Hadronization reduces the fiducial cross section by a few percents for HERWIG7. From table 7 we find normalizations of MC predictions generally lie between NLO predictions with and without corrections in top-quark decay. On the other hand, for the average $p_{T}, \mathrm{MC}$ predictions are closer to the NNLO predictions. The MC predictions on the average $p_{T}$ from different parton showers show a spread of $0.2 \sim 0.4 \mathrm{GeV}$ due to different approximations used for higher-order QCD corrections. That is not surprising since the NLO QCD corrections from top quark decay alone can induce a shift of similar size. We note that there exist MC generators including full NLO QCD corrections $[49,51]$ which will be discussed in the following section. In the future once a calculation at NNLO matched with parton shower becomes available we expect the dependence on parton showers can be largely reduced.

\section{Discussions}

In this section we further discuss several theory and experimental subjects which are relevant for extraction of the top-quark mass. That includes impact of various experimental selections, for example, contributions from leptonic decay of $\tau$ lepton in top-quark decay, isolation of lepton from jets, and $b$-tagging efficiency. Theory topics include contributions of non-resonant diagrams, non-factorized corrections, and electroweak corrections. In addition, we estimate various standard model backgrounds and propose a possible solution 


\begin{tabular}{|l|cc|cc|}
\hline \multirow{2}{*}{$\begin{array}{l}\left\langle p_{T, l}\right\rangle \\
{[\mathrm{GeV}]}\end{array}$} & \multicolumn{2}{|c}{ CMS-SA } & \multicolumn{2}{c|}{ CMS-SB } \\
\cline { 2 - 5 } & $<100 \mathrm{GeV}$ & $<200 \mathrm{GeV}$ & $<100 \mathrm{GeV}$ & $<200 \mathrm{GeV}$ \\
\hline PYTHIA8 & $47.66(47.69)$ & $50.05(50.06)$ & $47.43(47.47)$ & $49.25(49.30)$ \\
\hline PYTHIA6 & $47.54(47.51)$ & $50.01(49.97)$ & $47.23(47.24)$ & $49.15(49.18)$ \\
\hline HERWIG7 & $47.40(47.38)$ & $49.77(49.66)$ & $47.09(47.14)$ & $48.90(48.89)$ \\
\hline NNLO & 47.65 & 50.10 & 47.35 & 49.25 \\
\hline NLO(w/o decay) & $47.78(48.06)$ & $50.37(50.67)$ & $47.49(47.84)$ & $49.66(50.02)$ \\
\hline MCFM(w/o decay) & $47.81(48.08)$ & $50.40(50.70)$ & $47.52(47.87)$ & $49.69(50.04)$ \\
\hline
\end{tabular}

Table 6. Average transverse momentum of the charged lepton within the two fiducial regions, comparing predictions at fixed-order and those from various event generators in the 5FS for LHC $13 \mathrm{TeV}$. Numbers in parenthesis correspond to MC predictions without including hadronization or fixed-order predictions without including QCD corrections in decay of top quark.

\begin{tabular}{|l|cc|cc|}
\hline \multirow{2}{*}{\begin{tabular}{l}
$\sigma_{\text {fid. }}[\mathrm{pb}]$ \\
\cline { 2 - 5 }
\end{tabular}} & \multicolumn{2}{|c}{ CMS-SA } & \multicolumn{2}{c|}{ CMS-SB } \\
\hline PYTHIA8 & $<100 \mathrm{GeV}$ & $<200 \mathrm{GeV}$ & $<100 \mathrm{GeV}$ & $<200 \mathrm{GeV}$ \\
\hline PYTHIA6 & $1.23(1.24)$ & $1.27(1.28)$ & $0.565(0.570)$ & $0.580(0.580)$ \\
\hline HERWIG7 & $1.14(1.17)$ & $1.17(1.21)$ & $0.510(0.524)$ & $0.535(0.548)$ \\
\hline NNLO & 1.10 & 1.14 & 0.493 & 0.506 \\
\hline NLO(w/o decay) & $1.17(1.31)$ & $1.22(1.36)$ & $0.545(0.613)$ & $0.562(0.632)$ \\
\hline
\end{tabular}

Table 7. Fiducial cross sections within the two fiducial regions, comparing predictions at fixedorder and those from various event generators in the 5FS for LHC $13 \mathrm{TeV}$. Numbers in parenthesis correspond to $\mathrm{MC}$ predictions without including hadronization or fixed-order predictions without including QCD corrections in decay of top quark.

on reducing their impact. Results shown here are calculated with MG5 at leading order matched with parton shower and hadronization via PYTHIA6 unless otherwise specified.

\subsection{Signal selection and corrections}

We start with contributions from leptonic decay of $\tau$ lepton. They can be counted as either part of the signals or a background to be subtracted. The inclusive cross sections from $\tau$ decay are suppressed by a branching ratio of $17 \%$. In the fiducial regions selected, the $\tau$ contributions are further suppressed due to the $p_{T}$ threshold of charged lepton as well as the cut on transverse mass, since more neutrinos are presented in final state. For the same reason it has a softer spectrum for the charged lepton comparing to those from direct production. As shown in table 8 , the $\tau$ contributions amount to about $2 \%$ of the direct contributions for the fiducial cross sections, and reduce the average $p_{T}$ of charged lepton by $0.1 \mathrm{GeV}$.

In previous calculations we have not applied any isolation cuts on the charged lepton from jets, which are usually imposed in experimental analyses. We repeat our NLO cal- 


\begin{tabular}{|l|c|cc|cc|}
\hline \multirow{2}{*}[\mathrm{GeV}]{$/[\mathrm{pb}]$} & & \multicolumn{2}{|c}{ CMS-SA } & \multicolumn{2}{c|}{ CMS-SB } \\
\cline { 3 - 6 } & & $<100 \mathrm{GeV}$ & $<200 \mathrm{GeV}$ & $<100 \mathrm{GeV}$ & $<200 \mathrm{GeV}$ \\
\hline \multirow{2}{*}{$\tau$ decay } & $\delta\left\langle p_{T, l}\right\rangle$ & -0.13 & -0.15 & -0.13 & -0.15 \\
& $\delta \sigma_{f i d}$ & 0.026 & 0.026 & 0.012 & 0.012 \\
\hline \multirow{2}{*}{ lepton isolation } & $\delta\left\langle p_{T, l}\right\rangle$ & 0.10 & 0.10 & 0.05 & 0.06 \\
& $\delta \sigma_{f i d}$ & -0.017 & -0.017 & -0.003 & -0.003 \\
\hline \multirow{2}{*}{ b-tagging $(40 \%)$} & $\delta\left\langle p_{T, l}\right\rangle$ & 0.08 & 0.13 & 0.005 & 0.007 \\
\hline \multirow{2}{*}{ non-resonant } & $\delta\left\langle p_{T, l}\right\rangle$ & 0.15 & 0.26 & 0.02 & 0.06 \\
& $\delta \sigma_{\text {fid }}$ & -0.018 & -0.018 & 0.008 & 0.008 \\
\hline
\end{tabular}

Table 8. Changes of the average transverse momentum of the charged lepton and of the fiducial cross section within the two fiducial regions, when including contributions from $\tau$ decay, applying lepton isolation, varying $b$-tagging efficiency, and including non-resonant contributions.

culations by further requiring $\Delta R_{l j(b)}>0.4$. The changes on fiducial cross section and average $p_{T}$ are summarized in table 8 . The isolation cut has less impact when requiring the light jet in forward region, i.e., for the signal region CMS-SB, since then the charged lepton is unlikely to be close to the light jet. We also vary the $b$-tagging efficiency from our nominal choice of $50 \%$ to $40 \%$. That leads to an overall rescaling of the cross section and distributions except if there exist more than one true $b$-jets in the final state, which is the case for beyond leading order. By repeating our NLO calculations we found the changes on average $p_{T}$ is negligible for the signal region CMS-SB since it is unlikely the light jet is due to mistagging of true $b$-jet. In reality nonuniformity of $b$-tagging efficiency may lead to further changes of the average $p_{T}$ due to correlations between kinematics of the $b$ quark and of the charged lepton.

Next we move to various theory aspects starting with non-resonant contributions, namely production of $W^{+} b j$ via electroweak interactions without a top-quark resonance. Those non-resonant diagrams can interfere with the resonant diagrams and induce nonnegligible contributions as shown in table 8. The effects are much smaller in the fiducial region CMS-SB due to non-forward nature of the light jet in non-resonant production. We should mention that there are also non-resonant diagrams of $W^{+} b j$ production from QCD interactions. They do not interfere with the others at $\mathrm{LO}$ and we leave them to the $W^{+} J J$ category that will be discussed later in the background section.

\subsection{Non-factorized and EW corrections}

The NNLO predictions presented are based on a calculation using NWA and structure function approach. There exist missing QCD corrections due to non-factorized diagrams starting at NLO, e.g., with a gluon connecting bottom quarks in production and in decay of the top quark. Those non-factorized corrections have been studied in details in $[45,50]$. They are formally of the size $\alpha_{S} \Gamma_{t} / m_{t}$, namely suppressed by the width of the top quark, but can be enhanced in certain kinematic region. We follow the strategy in ref. [51] on 

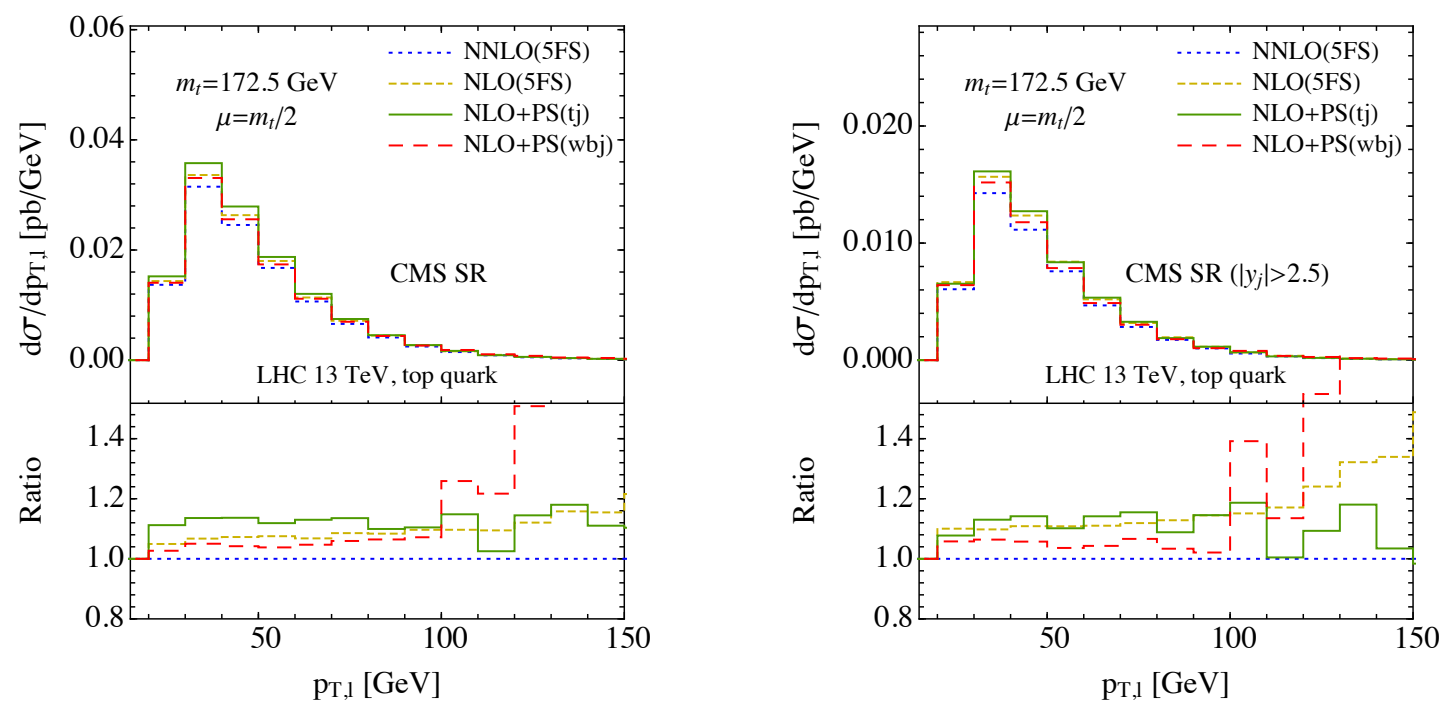

Figure 6. Transverse momentum distribution of the charged lepton within the two fiducial regions, comparing predictions at fixed-order and those from event generators $t j$ and $W b j$ (see text for details) in the $5 \mathrm{FS}$ for LHC $13 \mathrm{TeV}$.

identifying the corrections. We calculate the full NLO predictions for the production of final state $W^{+} b j$ and then subtract the contributions due to $s$-channel single top production and associated production of $t W$. All contributions are calculated using MG5_aMC@NLO with parton shower and hadronization applied from PYTHIA6, instead of calculated at fixed order.

The resulting transverse momentum distributions of the charged lepton are presented in figure 6, comparing with fixed-order results and MC result with PYTHIA6 shown in early sections. The two MC predictions are denoted as $t j$ and $W^{+} b j$ respectively. In comparison the latter includes exact NLO corrections in decay of the top quark and further the nonfactorized corrections just mentioned. For transverse momentum below $100 \mathrm{GeV}$ we find the $W^{+} b j$ predictions locate well in between previous NLO and NNLO fixed-order predictions. We find a trend of large enhancement of the distribution at beyond $100 \mathrm{GeV}$ in $W^{+} b j$ predictions though accompanied with significant MC statistical errors. In ref. [51] it also shows the NLO electroweak corrections can induce a significant change on shapes of various distributions. For comparison to experimental data, a recalculation of the full NLO QCD and EW corrections focusing on transverse momentum of the charged lepton will be desirable. That can be done following various techniques outlined in ref. [51] and with a careful separation of backgrounds that are already accounted for in the experimental analyses.

Finally there are also non-factorized NNLO QCD corrections in production stage which are beyond the structure function approach, for example, from the double-box diagrams and also interferences of $t$-channel and $s$-channel at NNLO. It is not clear how they may change shape of various distributions. Giving the fact that they are suppressed by QCD colors and also considering the size of the known NNLO corrections in production, we estimate 
their impact to the average $p_{T}$ to be within the scale variations considered. Similar nonfactorized corrections also exist in production of the Higgs boson and are estimated using eikonal approximation [101, 102].

\subsection{Backgrounds}

The SM backgrounds mainly consist of top-quark pair production, single top-quark production in $s$-channel and in associated with a $W$ boson, QCD production of $W J J$, and diboson production. For QCD production of $W J J$, the jet $J$ can arise from not only a bottom quark, but also a charm quark, even a gluon or a light quark. In the latter it mimics the signal due to mistagging which we choose a rate of $3 \%$ for charm quark and $0.1 \%$ for gluon and light quarks [74]. We summarize the fiducial cross sections of various backgrounds and the average transverse momentum in table 9. For comparison we also show backgrounds for top anti-quark production, and include numbers for signal process as well which are calculated at NNLO. The average momentums are calculated from spectrums of individual signals and backgrounds in the fiducial regions. They are close in size for signal and backgrounds due to the same kinematic selections used, for instance, a threshold of $26 \mathrm{GeV}$ on $p_{T}$ of the charged lepton. In table 9 we do not repeat the row if the background contributes equally to top quark and anti-quark processes.

In general the top-quark pair production is dominant among all backgrounds due to the large cross section, though it requires the additional charged lepton or jets lie outside the acceptance region. We veto any event with more than one charged lepton with $p_{T}>$ $10 \mathrm{GeV}$. The primary charged lepton has larger transverse momentum in pair production due to the relatively large $p_{T}$ of the top-quark. Large contributions are also seen for QCD production of $W J J$ which has a harder $p_{T}$ spectrum for the charged lepton. The $t W$ associated production can contribute at a level of tens percents of the signal cross sections. We note for signal of top-quark production, both $t W^{-}$and $\bar{t} W^{+}$production can contribute as backgrounds. In the latter case the primary charged lepton comes directly from $W^{+}$ decay leading to a harder $p_{T}$ spectrum. Backgrounds due to $s$-channel production or diboson production are small. A typical feature can be seen from table 9 is that in signal region CMS-SB where the light jet is required to be forward, almost all backgrounds are suppressed by a factor of ten at least. The signal from $t$-channel production are less affected due to the forward nature of the light jet.

From table 9 we find even in the region CMS-SB, the rate of $t \bar{t}$ background can still reach the same level as the signal processes. That can easily spoil the precision on measurement of the average $p_{T}$ for the signal processes due to uncertainties on modeling of the $t \bar{t}$ background. Further more, any backgrounds from top-quark production depend on the top-quark mass as well, which will complicate the extraction of the top-quark mass. One important observation is that both the $t \bar{t}$ and $t W$ backgrounds contribute almost equally to signal processes of charged lepton with positive and negative charges. The charge asymmetry first enters at NLO for $t \bar{t}$ production and is small at the LHC. In case of $t W$ production the asymmetry vanishes even at NLO. Thus one possibility is to measure the difference of lepton $p_{T}$ spectrums for positive and negative charges. Dependence and associated uncertainties on modeling of the $t \bar{t}$ and $t W$ backgrounds are minimized, though their statistical 


\begin{tabular}{|c|c|c|c|c|c|}
\hline \multirow[t]{2}{*}[\mathrm{GeV}]{$/[\mathrm{pb}]$} & & \multicolumn{2}{|c|}{ CMS-SA } & \multicolumn{2}{|c|}{ CMS-SB } \\
\hline & & $<100 \mathrm{GeV}$ & $<200 \mathrm{GeV}$ & $<100 \mathrm{GeV}$ & $<200 \mathrm{GeV}$ \\
\hline \multirow{2}{*}{$t \bar{t}$} & $\left\langle p_{T, l}\right\rangle$ & 52.2 & 59.8 & 51.9 & 59.1 \\
\hline & $\sigma_{f i d}$ & 4.42 & 4.93 & 0.40 & 0.44 \\
\hline \multirow{2}{*}{$t W^{-}\left(\bar{t} W^{+}\right)$} & $\left\langle p_{T, l}\right\rangle$ & 52.2 & 61.8 & 52.5 & 61.1 \\
\hline & $\sigma_{f i d}$ & 0.33 & 0.38 & 0.019 & 0.021 \\
\hline \multirow{2}{*}{$s$-channel $t$} & $\left\langle p_{T, l}\right\rangle$ & 47.6 & 50.6 & 47.2 & 49.4 \\
\hline & $\sigma_{f i d}$ & 0.044 & 0.046 & 0.007 & 0.007 \\
\hline \multirow{2}{*}{$s$-channel $\bar{t}$} & $\left\langle p_{T, l}\right\rangle$ & 47.7 & 50.3 & 47.4 & 49.1 \\
\hline & $\sigma_{f i d}$ & 0.030 & 0.031 & 0.004 & 0.004 \\
\hline \multirow{2}{*}{$\mathrm{QCD} W^{+} J J$} & $\left\langle p_{T, l}\right\rangle$ & 50.5 & 59.2 & 51.0 & 58.8 \\
\hline & $\sigma_{f i d}$ & 1.29 & 1.45 & 0.157 & 0.174 \\
\hline \multirow{2}{*}{ QCD $W^{-} J J$} & $\left\langle p_{T, l}\right\rangle$ & 52.5 & 64.2 & 52.9 & 62.8 \\
\hline & $\sigma_{f i d}$ & 0.99 & 1.15 & 0.107 & 0.117 \\
\hline \multirow{2}{*}{$W^{+} Z$} & $\left\langle p_{T, l}\right\rangle$ & 53.0 & 65.1 & 55.2 & 68.5 \\
\hline & $\sigma_{f i d}$ & 0.005 & 0.006 & 0.0008 & 0.0009 \\
\hline \multirow{2}{*}{$W^{-} Z$} & $\left\langle p_{T, l}\right\rangle$ & 52.7 & 63.5 & 51.8 & 60.2 \\
\hline & $\sigma_{f i d}$ & 0.004 & 0.004 & 0.0005 & 0.0006 \\
\hline \multirow{2}{*}{$t$-channel $t$} & $\left\langle p_{T, l}\right\rangle$ & 47.65 & 50.10 & 47.35 & 49.25 \\
\hline & $\sigma_{f i d}$ & 1.10 & 1.14 & 0.493 & 0.506 \\
\hline \multirow{2}{*}{$t$-channel $\bar{t}$} & $\left\langle p_{T, l}\right\rangle$ & 47.85 & 50.17 & 47.70 & 49.54 \\
\hline & $\sigma_{f i d}$ & 0.674 & 0.696 & 0.250 & 0.257 \\
\hline
\end{tabular}

Table 9. Average transverse momentum of the charged lepton and fiducial cross section within the two fiducial regions, for various background processes to $t$-channel top quark and anti-quark production. The top-quark pair production or top-quark associated production with $W$ boson contribute equally to the two charge conjugate final states.

fluctuations remain. Sensitivity of the signal processes to top-quark mass and the theoretical uncertainties are almost unchanged when taking differences of spectrums with opposite charges. That is because the differences of $t$-channel single top quark and anti-quark production are mostly driven by different parton distributions at the light-quark line. At the end, the uncertainties due to modeling of QCD WJJ background will be dominant. However, as mentioned earlier, a large fraction of $W J J$ background arise from production of charm quark, gluon or light quarks which are misidentified as $b$-jets. One can further reduce their impact by either imposing a tighter $b$-tagging criteria or using data-driven methods. 


\section{Projection for (HL-)LHC}

We provide an estimation on precision of the top-quark mass measurement can be achieved in the coming run of LHC and HL-LHC. As explained earlier, the observable used is the average transverse momentum of the charged lepton in the charge-weighted distribution,

$$
\begin{aligned}
\left\langle p_{T}\right\rangle_{\mathrm{obs}} & \equiv \frac{\int p_{T, l}\left[d \sigma^{l^{+}}-d \sigma^{l^{-}}\right]}{\sigma^{l^{+}}-\sigma^{l^{-}}} \\
& =\frac{\int p_{T, l}\left[d \sigma_{S}^{l^{+}}-d \sigma_{S}^{l^{-}}+d \sigma_{B}^{l^{+}}-d \sigma_{B}^{l^{-}}\right]}{\sigma_{S}^{l^{+}}-\sigma_{S}^{l^{-}}+\sigma_{B}^{l^{+}}-\sigma_{B}^{l^{-}}} \\
& =\left\langle p_{T}\right\rangle_{S}+\frac{r}{1+r}\left[\left\langle p_{T}\right\rangle_{B}-\left\langle p_{T}\right\rangle_{S}\right],
\end{aligned}
$$

where in the second line we have rewritten the average $p_{T}$ in terms of signal and background contributions. $\left\langle p_{T}\right\rangle_{S(B)}$ are the average $p_{T}$ of the charged lepton in the spectrum of signal(background) alone,

$$
\left\langle p_{T}\right\rangle_{S(B)} \equiv \frac{\int p_{T, l}\left[d \sigma_{S(B)}^{l^{+}}-d \sigma_{S(B)}^{l^{-}}\right]}{\sigma_{S(B)}^{l^{+}}-\sigma_{S(B)}^{l^{-}}},
$$

and $r$ is the background to signal ratio,

$$
r \equiv \frac{\sigma_{B}^{l^{+}}-\sigma_{B}^{l^{-}}}{\sigma_{S}^{l^{+}}-\sigma_{S}^{l^{-}}}
$$

We neglect backgrounds other than from top-quark pair production and QCD production of $W J J$, which are small according to table 9. From eq. (6.1) we can extract the average transverse momentum of the signal $\left\langle p_{T}\right\rangle_{S}$ using the measurement on $\left\langle p_{T}\right\rangle_{\text {obs }}$ and inputs of $r$ and $\left\langle p_{T}\right\rangle_{B}$. From our theory calculation we can arrive at a linear model on dependence of the average $p_{T}$ on the top-quark mass,

$$
\left\langle p_{T}\right\rangle_{S}=p_{T, 0}+\lambda\left[\frac{m_{t}}{\mathrm{GeV}}-172.5\right],
$$

where $p_{T, 0}$ is the average $p_{T}$ of signal for a top-quark mass of $172.5 \mathrm{GeV} \cdot p_{T, 0}$ and $\lambda$ can be derived from NNLO predictions shown in tables 1-3 together with the counterparts for top anti-quark production. By combining eqs. (6.1) and (6.4) we can extract the top-quark mass.

In the following we focus on the signal region CMS-SB with $p_{T, l}<100 \mathrm{GeV}$. It benefits from both lower backgrounds and smaller theoretical uncertainties. We estimate several contributions to the final uncertainty of measured top-quark mass. The statistical uncertainty on $\left\langle p_{T}\right\rangle_{\text {obs }}$ due to fluctuations of both signal and backgrounds, including $t \bar{t}$ contributions, are computed with pseudo experiment assuming an integrated luminosity of 300 and $3000 \mathrm{fb}^{-1}$ respectively and assuming top-quark decays into two families of leptons. Theoretical uncertainties on $p_{T, 0}$ are estimated with scale variations of the NNLO predictions shown in table 1. In figure 7 we plot results on determination of the top-quark mass with a hypothetical value of $172.5 \mathrm{GeV}$. The horizontal bands indicate the statistical uncertainties 


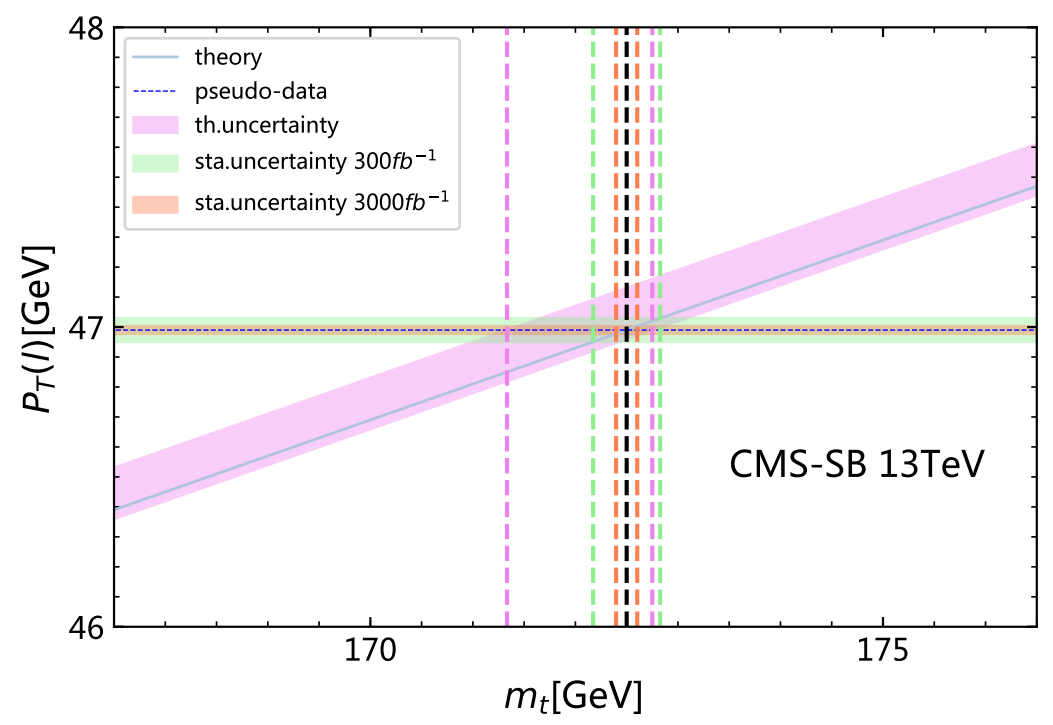

Figure 7. Predictions on the average transverse momentum of the charged lepton in the signal process as a function of the top-quark mass (band along diagonal direction) and the projected measurement on the same quantity with only statistical errors (horizontal bands). Extracted topquark mass with various uncertainties are indicated by vertical lines.

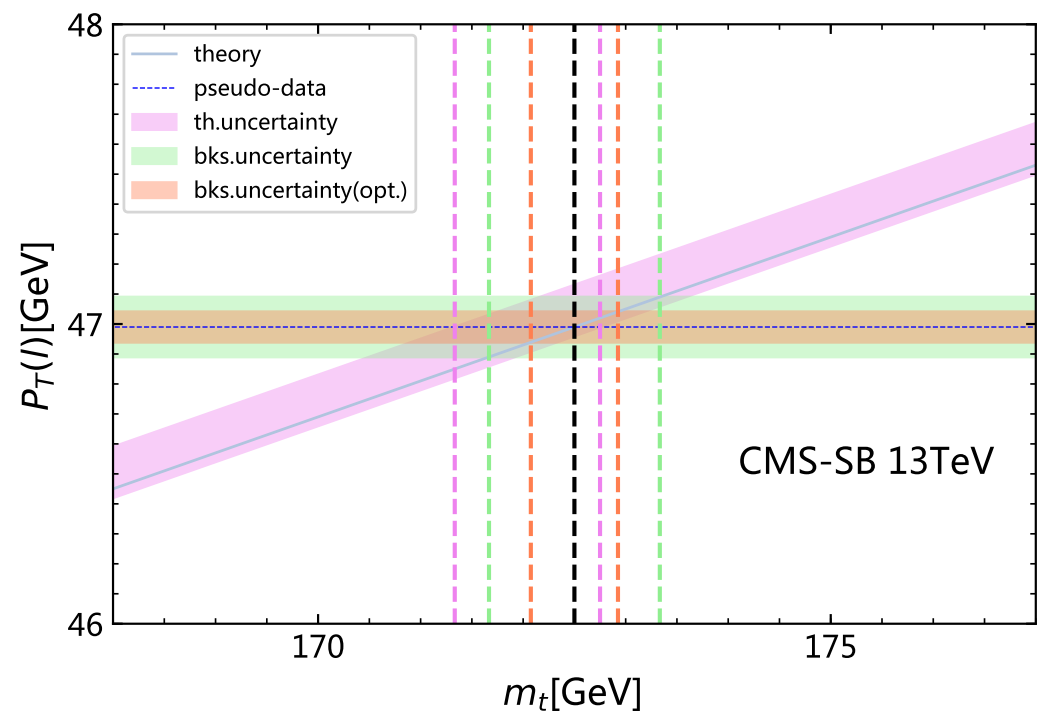

Figure 8. Predictions on the average transverse momentum of the charged lepton of the signal process as a function of the top-quark mass (band along diagonal direction) and the projected measurement on the same quantity with only systematic errors from background modeling (horizontal bands). Extracted top-quark mass with various uncertainties are indicated by vertical lines. 
as propagated into $\left\langle p_{T}\right\rangle_{S}$. The diagonal band represents the theory prediction of $\left\langle p_{T}\right\rangle_{S}$ as a function of the top-quark mass including scale variations. The projected uncertainties on the extracted top-quark mass are computed assuming linear error propagation, and are represented by vertical lines. For example, the statistical uncertainty is about $\pm 0.3(0.1) \mathrm{GeV}$ with an integrated luminosity of $300(3000) \mathrm{fb}^{-1}$. The theoretical uncertainty amounts to +0.3 and $-1.2 \mathrm{GeV}$.

Further uncertainties are related to modeling of the backgrounds. We only need to consider the $W J J$ background in this case since the systematic uncertainty for $t \bar{t}$ production cancels in the charge weighted $p_{T}$ distribution. A precise study on QCD $W J J$ background is beyond the scope of current paper, and can be carried out with dedicated MC simulations. We simply assign empirical numbers on systematic uncertainties of $\left\langle p_{T}\right\rangle_{B}$ and $r$ from $W J J$ background. On one hand we assume they are $0.5 \mathrm{GeV}$ and $10 \%$ respectively, and reduced by a factor of two in the optimistic case. The results are shown in figure 8 with the horizontal bands representing uncertainty of $\left\langle p_{T}\right\rangle_{S}$ as propagated from systematic errors of backgrounds. The uncertainty on measured top-quark mass is 0.8 and $0.4 \mathrm{GeV}$ for the two scenarios respectively as shown by vertical lines in figure 8, comparing to the theoretical uncertainty shown earlier. Thus we expect the full error budget of the extracted topquark mass consists of a theoretical uncertainty of about $1 \mathrm{GeV}$ from signal modeling, a systematic uncertainty of $0.4 \mathrm{GeV}$ due to background modeling, and a much smaller statistical uncertainty.

\section{Summary}

In summary we have studied the determination of the top-quark mass using leptonic observables in $t$-channel single top-quark production at the LHC. Extraction of the top-quark mass from single top-quark production benefits from the fact that systematic uncertainties are partially uncorrelated to those in top-quark pair production on both experimental and theory sides. We demonstrate sensitivity of the average transverse momentum of the charged lepton to the top-quark mass. Leptonic observables are generally believed to be less affected by various non-perturbative QCD effects and the jet energy scale uncertainties. We identify an appropriate signal region for such a measurement at the LHC with enhanced signal to background ratio as well as stable theory predictions.

We present our NNLO QCD predictions under narrow width approximation using structure function approach. We show that QCD corrections in top-quark decay play important role for such leptonic observables. We find a good convergence on predictions of the average transverse momentum of the charged lepton with scale uncertainties well under control. By comparing our fixed-order predictions to predictions from MC generators we find the parton shower resummation can capture part of the NLO and NNLO corrections, and the hadronization effects are in general small for leptonic observables. Besides, we point out several corrections that need to be included when comparing our NNLO predictions with data, including non-resonant corrections, non-factorized QCD corrections, EW corrections, and so on. 
Moreover, we estimate various SM backgrounds to the signals considered. We propose to use the charge weighted distribution in the measurement, i.e., difference between distributions of charged lepton with positive and negative electric charges. That can reduce uncertainties due to modeling of SM backgrounds which contribute equally to final states with different charges, for example, backgrounds from QCD jets production, top-quark pair production, and top-quark associated production with a $\mathrm{W}$ boson. We construct a simple model on dependence of the observed average transverse momentum of the charged lepton to the top quark mass, and present projections for future (HL-)LHC measurement on top quark mass. The statistical uncertainties and theoretical uncertainties due to hadronization corrections are found to be small. Scale variations in our signal modeling transfer into an uncertainty of about $1 \mathrm{GeV}$ on the extracted top-quark mass. However, the scale variations should be considered as an optimistic estimation on the uncertainty due to the missing higher-order corrections. Future works on the unknown non-factorized corrections as well as on matching NNLO calculations with parton showers can provide a better understanding of the perturbative uncertainties. Lastly theoretical uncertainty due to modeling of remaining SM backgrounds is estimated to be $0.4 \sim 0.8 \mathrm{GeV}$.

\section{Acknowledgments}

The work of J. Gao was sponsored by the National Natural Science Foundation of China under the Grant No. 11875189 and No.11835005. The authors would like to thank Kai Yan and Dingyu Shao for proofreading of the manuscript.

Open Access. This article is distributed under the terms of the Creative Commons Attribution License (CC-BY 4.0), which permits any use, distribution and reproduction in any medium, provided the original author(s) and source are credited.

\section{References}

[1] J. Haller, A. Hoecker, R. Kogler, K. Mönig, T. Peiffer and J. Stelzer, Update of the global electroweak fit and constraints on two-Higgs-doublet models, Eur. Phys. J. C 78 (2018) 675 [arXiv: 1803. 01853] [INSPIRE].

[2] G. Isidori, G. Ridolfi and A. Strumia, On the metastability of the standard model vacuum, Nucl. Phys. B 609 (2001) 387 [hep-ph/0104016] [INSPIRE].

[3] CDF and D0 collaborations, Combination of CDF and D0 results on the mass of the top quark using up to $9.7 \mathrm{fb}^{-1}$ at the Tevatron, arXiv:1407.2682 [INSPIRE].

[4] ATLAS collaboration, Measurement of the top quark mass in the $t \bar{t} \rightarrow$ lepton + jets channel from $\sqrt{s}=8 \mathrm{TeV}$ ATLAS data and combination with previous results, Eur. Phys. J. C 79 (2019) 290 [arXiv: 1810.01772] [INSPIRE].

[5] CMS collaboration, Measurement of the top quark mass in the all-jets final state at $\sqrt{s}=13 \mathrm{TeV}$ and combination with the lepton+jets channel, Eur. Phys. J. C 79 (2019) 313 [arXiv: 1812.10534] [INSPIRE].

[6] A.H. Hoang, The top mass: interpretation and theoretical uncertainties, in $7^{\text {th }}$ international workshop on top quark physics, (2014) [arXiv:1412.3649] [INSPIRE]. 
[7] P. Nason, The top mass in hadronic collisions, in From my vast repertoire...: Guido Altarelli's legacy, A. Levy, S. Forte and G. Ridolfi eds., World Scientific, Singapore (2019) [arXiv: 1712.02796] [INSPIRE].

[8] A.H. Hoang, S. Plätzer and D. Samitz, On the cutoff dependence of the quark mass parameter in angular ordered parton showers, JHEP 10 (2018) 200 [arXiv:1807.06617] [INSPIRE].

[9] S. Ferrario Ravasio, Top-mass observables: all-orders behaviour, renormalons and $\mathrm{NLO}+$ parton shower effects, Ph.D. thesis, Milan Bicocca U., Milan, Italy (2018) [arXiv: 1902.05035] [INSPIRE].

[10] S. Ferrario Ravasio, T. Ježo, P. Nason and C. Oleari, A theoretical study of top-mass measurements at the LHC using $N L O+P S$ generators of increasing accuracy, Eur. Phys. J. C 78 (2018) 458 [Addendum ibid. 79 (2019) 859] [arXiv: 1906.09166] [INSPIRE].

[11] M. Beneke, P. Marquard, P. Nason and M. Steinhauser, On the ultimate uncertainty of the top quark pole mass, Phys. Lett. B $\mathbf{7 7 5}$ (2017) 63 [arXiv: 1605.03609] [InSPIRE].

[12] A.H. Hoang et al., The MSR mass and the $\mathcal{O}\left(\Lambda_{\mathrm{QCD}}\right)$ renormalon sum rule, JHEP 04 (2018) 003 [arXiv: 1704.01580] [inSPIRE].

[13] A.H. Hoang, C. Lepenik and M. Preisser, On the light massive flavor dependence of the large order asymptotic behavior and the ambiguity of the pole mass, JHEP 09 (2017) 099 [arXiv: 1706.08526] [INSPIRE].

[14] C.G. Lester and D.J. Summers, Measuring masses of semiinvisibly decaying particles pair produced at hadron colliders, Phys. Lett. B 463 (1999) 99 [hep-ph/9906349] [INSPIRE].

[15] S. Frixione and A. Mitov, Determination of the top quark mass from leptonic observables, JHEP 09 (2014) 012 [arXiv:1407.2763] [INSPIRE].

[16] K. Agashe, R. Franceschini, D. Kim and M. Schulze, Top quark mass determination from the energy peaks of b-jets and B-hadrons at NLO QCD, Eur. Phys. J. C 76 (2016) 636 [arXiv: 1603.03445] [INSPIRE].

[17] CMS collaboration, Measurement of the top quark mass using charged particles in pp collisions at $\sqrt{s}=8 \mathrm{TeV}$, Phys. Rev. D 93 (2016) 092006 [arXiv:1603.06536] [InSPIRE].

[18] CMS collaboration, Measurement of the mass of the top quark in decays with a $J / \psi$ meson in pp collisions at $8 \mathrm{TeV}$, JHEP 12 (2016) 123 [arXiv: 1608.03560] [INSPIRE].

[19] ATLAS collaboration, Measurement of lepton differential distributions and the top quark mass in $t \bar{t}$ production in pp collisions at $\sqrt{s}=8 \mathrm{TeV}$ with the ATLAS detector, Eur. Phys. J. $C 77$ (2017) 804 [arXiv: 1709.09407] [InSPIRE].

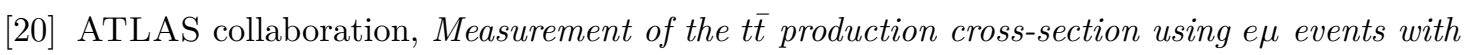
b-tagged jets in pp collisions at $\sqrt{s}=7$ and $8 \mathrm{TeV}$ with the ATLAS detector, Eur. Phys. J. C 74 (2014) 3109 [Addendum ibid. 76 (2016) 642] [arXiv: 1406.5375] [INSPIRE].

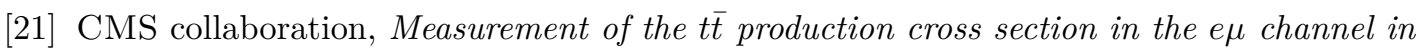
proton-proton collisions at $\sqrt{s}=7$ and $8 \mathrm{TeV}$, JHEP 08 (2016) 029 [arXiv:1603.02303] [INSPIRE].

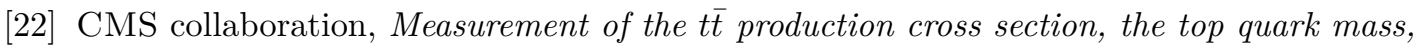
and the strong coupling constant using dilepton events in pp collisions at $\sqrt{s}=13 \mathrm{TeV}$, Eur. Phys. J. C 79 (2019) 368 [arXiv:1812.10505] [InSPIRE]. 
[23] CMS collaboration, Measurement of t $\bar{t}$ normalised multi-differential cross sections in $p p$ collisions at $\sqrt{s}=13 \mathrm{TeV}$, and simultaneous determination of the strong coupling strength, top quark pole mass, and parton distribution functions, Eur. Phys. J. C 80 (2020) 658 [arXiv: 1904.05237] [INSPIRE].

[24] W.-L. Ju, G. Wang, X. Wang, X. Xu, Y. Xu and L.L. Yang, Invariant-mass distribution of top-quark pairs and top-quark mass determination, Chin. Phys. C 44 (2020) 091001 [arXiv: 1908.02179] [INSPIRE].

[25] W.-L. Ju, G. Wang, X. Wang, X. Xu, Y. Xu and L.L. Yang, Top quark pair production near threshold: single/double distributions and mass determination, JHEP 06 (2020) 158 [arXiv: 2004.03088] [INSPIRE].

[26] S. Alioli et al., A new observable to measure the top-quark mass at hadron colliders, Eur. Phys. J. C $\mathbf{7 3}$ (2013) 2438 [arXiv:1303.6415] [INSPIRE].

[27] ATLAS collaboration, Measurement of the top quark mass in topologies enhanced with single top quarks produced in the t-channel at $\sqrt{s}=8 \mathrm{TeV}$ using the ATLAS experiment, in $7^{\text {th }}$ international workshop on top quark physics, (2014) [arXiv:1411.3879] [INSPIRE].

[28] CMS collaboration, Measurement of the top quark mass using single top quark events in proton-proton collisions at $\sqrt{s}=8 \mathrm{TeV}$, Eur. Phys. J. C 77 (2017) 354 [arXiv:1703.02530] [INSPIRE].

[29] S. Alekhin, S. Moch and S. Thier, Determination of the top-quark mass from hadro-production of single top-quarks, Phys. Lett. B 763 (2016) 341 [arXiv:1608.05212] [INSPIRE].

[30] M. Beneke, Y. Kiyo, P. Marquard, A. Penin, J. Piclum and M. Steinhauser, Next-to-next-to-next-to-leading order QCD prediction for the top antitop S-wave pair production cross section near threshold in $e^{+} e^{-}$annihilation, Phys. Rev. Lett. 115 (2015) 192001 [arXiv: 1506.06864] [INSPIRE].

[31] A.H. Hoang, What is the top quark mass?, Ann. Rev. Nucl. Part. Sci. 70 (2020) 225 [arXiv: 2004.12915] [INSPIRE].

$[32]$ G. Bordes and B. van Eijk, Calculating QCD corrections to single top production in hadronic interactions, Nucl. Phys. B 435 (1995) 23 [InSPIRE].

[33] R. Pittau, Final state QCD corrections to off-shell single top production in hadron collisions, Phys. Lett. B 386 (1996) 397 [hep-ph/9603265] [INSPIRE].

[34] T. Stelzer, Z. Sullivan and S. Willenbrock, Single top quark production via $W$-gluon fusion at next-to-leading order, Phys. Rev. D 56 (1997) 5919 [hep-ph/9705398] [INSPIRE].

[35] T. Stelzer, Z. Sullivan and S. Willenbrock, Single top quark production at hadron colliders, Phys. Rev. D 58 (1998) 094021 [hep-ph/9807340] [INSPIRE].

[36] B.W. Harris, E. Laenen, L. Phaf, Z. Sullivan and S. Weinzierl, Fully differential QCD corrections to single top quark final states, Int. J. Mod. Phys. A 16S1A (2001) 379 [hep-ph/0102126] [INSPIRE].

[37] B.W. Harris, E. Laenen, L. Phaf, Z. Sullivan and S. Weinzierl, The fully differential single top quark cross-section in next to leading order QCD, Phys. Rev. D 66 (2002) 054024 [hep-ph/0207055] [INSPIRE].

[38] Z. Sullivan, Understanding single-top-quark production and jets at hadron colliders, Phys. Rev. D 70 (2004) 114012 [hep-ph/0408049] [INSPIRE]. 
[39] J.M. Campbell, R.K. Ellis and F. Tramontano, Single top production and decay at next-to-leading order, Phys. Rev. D 70 (2004) 094012 [hep-ph/0408158] [INSPIRE].

[40] Z. Sullivan, Angular correlations in single-top-quark and Wjj production at next-to-leading order, Phys. Rev. D 72 (2005) 094034 [hep-ph/0510224] [INSPIRE].

[41] Q.-H. Cao, R. Schwienhorst, J.A. Benitez, R. Brock and C.P. Yuan, Next-to-leading order corrections to single top quark production and decay at the Tevatron: $2 . t^{-}$channel process, Phys. Rev. D 72 (2005) 094027 [hep-ph/0504230] [INSPIRE].

[42] P. Falgari, P. Mellor and A. Signer, Production-decay interferences at NLO in QCD for t-channel single-top production, Phys. Rev. D 82 (2010) 054028 [arXiv:1007.0893] [INSPIRE].

[43] R. Schwienhorst, C.P. Yuan, C. Mueller and Q.-H. Cao, Single top quark production and decay in the t-channel at next-to-leading order at the LHC, Phys. Rev. D 83 (2011) 034019 [arXiv: 1012.5132] [INSPIRE].

[44] P. Falgari, F. Giannuzzi, P. Mellor and A. Signer, Off-shell effects for t-channel and s-channel single-top production at NLO in QCD, Phys. Rev. D 83 (2011) 094013 [arXiv:1102.5267] [INSPIRE].

[45] A.S. Papanastasiou, R. Frederix, S. Frixione, V. Hirschi and F. Maltoni, Single-top t-channel production with off-shell and non-resonant effects, Phys. Lett. B $\mathbf{7 2 6}$ (2013) 223 [arXiv:1305.7088] [INSPIRE].

[46] P. Kant et al., HatHor for single top-quark production: updated predictions and uncertainty estimates for single top-quark production in hadronic collisions, Comput. Phys. Commun. 191 (2015) 74 [arXiv:1406.4403] [INSPIRE].

[47] S. Carrazza, R. Frederix, K. Hamilton and G. Zanderighi, MINLO t-channel single-top plus jet, JHEP 09 (2018) 108 [arXiv: 1805.09855] [INSPIRE].

[48] J.M. Campbell, R. Frederix, F. Maltoni and F. Tramontano, Next-to-leading-order predictions for t-channel single-top production at hadron colliders, Phys. Rev. Lett. 102 (2009) 182003 [arXiv:0903.0005] [INSPIRE].

[49] R. Frederix, S. Frixione, A.S. Papanastasiou, S. Prestel and P. Torrielli, Off-shell single-top production at NLO matched to parton showers, JHEP 06 (2016) 027 [arXiv:1603.01178] [INSPIRE].

[50] T. Neumann and Z.E. Sullivan, Off-shell single-top-quark production in the standard model effective field theory, JHEP 06 (2019) 022 [arXiv:1903.11023] [INSPIRE].

[51] R. Frederix, D. Pagani and I. Tsinikos, Precise predictions for single-top production: the impact of EW corrections and QCD shower on the t-channel signature, JHEP 09 (2019) 122 [arXiv: 1907.12586] [INSPIRE].

[52] J. Wang, C.S. Li, H.X. Zhu and J.J. Zhang, Factorization and resummation of t-channel single top quark production, arXiv:1010.4509 [INSPIRE].

[53] N. Kidonakis, Next-to-next-to-leading-order collinear and soft gluon corrections for t-channel single top quark production, Phys. Rev. D 83 (2011) 091503 [arXiv:1103.2792] [INSPIRE].

[54] J. Wang, C.S. Li and H.X. Zhu, Resummation prediction on top quark transverse momentum distribution at large $p_{T}$, Phys. Rev. D 87 (2013) 034030 [arXiv:1210.7698] [INSPIRE]. 
[55] N. Kidonakis, Single-top transverse-momentum distributions at approximate NNLO, Phys. Rev. D 93 (2016) 054022 [arXiv: 1510.06361] [INSPIRE].

[56] Q.-H. Cao, P. Sun, B. Yan, C.P. Yuan and F. Yuan, Transverse momentum resummation for t-channel single top quark production at the LHC, Phys. Rev. D 98 (2018) 054032 [arXiv: 1801.09656] [INSPIRE].

[57] N. Kidonakis, Soft anomalous dimensions for single-top production at three loops, Phys. Rev. D 99 (2019) 074024 [arXiv: 1901.09928] [InSPIRE].

[58] Q.-H. Cao, P. Sun, B. Yan, C.P. Yuan and F. Yuan, Soft gluon resummation in t-channel single top quark production at the LHC, arXiv:1902.09336 [INSPIRE].

[59] S. Frixione, E. Laenen, P. Motylinski and B.R. Webber, Single-top production in MC@NLO, JHEP 03 (2006) 092 [hep-ph/0512250] [INSPIRE].

[60] S. Alioli, P. Nason, C. Oleari and E. Re, NLO single-top production matched with shower in POWHEG: s- and t-channel contributions, JHEP 09 (2009) 111 [Erratum ibid. 02 (2010) 011] [arXiv:0907.4076] [INSPIRE].

[61] R. Frederix, E. Re and P. Torrielli, Single-top t-channel hadroproduction in the four-flavour scheme with POWHEG and aMC@NLO, JHEP 09 (2012) 130 [arXiv:1207.5391] [INSPIRE].

[62] M. Brucherseifer, F. Caola and K. Melnikov, On the NNLO QCD corrections to single-top production at the LHC, Phys. Lett. B 736 (2014) 58 [arXiv:1404.7116] [INSPIRE].

[63] E.L. Berger, J. Gao, C.P. Yuan and H.X. Zhu, NNLO QCD corrections to t-channel single top-quark production and decay, Phys. Rev. D 94 (2016) 071501 [arXiv:1606.08463] [INSPIRE].

[64] E.L. Berger, J. Gao and H.X. Zhu, Differential distributions for $t$-channel single top-quark production and decay at next-to-next-to-leading order in QCD, JHEP 11 (2017) 158 [arXiv: 1708.09405] [INSPIRE].

[65] Z.L. Liu and J. Gao, s-channel single top quark production and decay at next-to-next-to-leading-order in QCD, Phys. Rev. D 98 (2018) 071501 [arXiv:1807.03835] [INSPIRE].

[66] J. Gao and E.L. Berger, Modeling of t-channel single top-quark production at the LHC, Phys. Lett. B $\mathbf{8 1 1}$ (2020) 135886 [arXiv:2005.12936] [INSPIRE].

[67] J. Lindfors, Higgs boson production by $W$ and $Z$ collisions, Phys. Lett. B 167 (1986) 471 [INSPIRE].

[68] T. Han, G. Valencia and S. Willenbrock, Structure function approach to vector boson scattering in pp collisions, Phys. Rev. Lett. 69 (1992) 3274 [hep-ph/9206246] [INSPIRE].

[69] T. Stelzer, Z. Sullivan and S. Willenbrock, Single top quark production via $W$-gluon fusion at next-to-leading order, Phys. Rev. D 56 (1997) 5919 [hep-ph/9705398] [INSPIRE].

[70] M. Assadsolimani, P. Kant, B. Tausk and P. Uwer, Calculation of two-loop QCD corrections for hadronic single top-quark production in the t-channel, Phys. Rev. D 90 (2014) 114024 [arXiv: 1409.3654] [INSPIRE].

[71] C. Meyer, Transforming differential equations of multi-loop Feynman integrals into canonical form, JHEP 04 (2017) 006 [arXiv: 1611.01087] [INSPIRE]. 
[72] A. Czarnecki and M. Jezabek, Distributions of leptons in decays of polarized heavy quarks, Nucl. Phys. B 427 (1994) 3 [hep-ph/9402326] [INSPIRE].

[73] Particle Data Group collaboration, Review of particle physics, Phys. Rev. D 98 (2018) 030001 [INSPIRE].

[74] CMS collaboration, Measurement of differential cross sections and charge ratios for $t$-channel single top quark production in proton-proton collisions at $\sqrt{s}=13 \mathrm{TeV}$, Eur. Phys. J. C 80 (2020) 370 [arXiv:1907.08330] [inSPIRE].

[75] M. Cacciari, G.P. Salam and G. Soyez, The anti- $k_{t}$ jet clustering algorithm, JHEP 04 (2008) 063 [arXiv:0802.1189] [InSPIRE].

[76] I.W. Stewart, F.J. Tackmann and W.J. Waalewijn, $N$-jettiness: an inclusive event shape to veto jets, Phys. Rev. Lett. 105 (2010) 092002 [arXiv: 1004.2489] [INSPIRE].

[77] R. Boughezal, C. Focke, X. Liu and F. Petriello, $W$-boson production in association with a jet at next-to-next-to-leading order in perturbative QCD, Phys. Rev. Lett. 115 (2015) 062002 [arXiv: 1504.02131] [INSPIRE].

[78] J. Gaunt, M. Stahlhofen, F.J. Tackmann and J.R. Walsh, $N$-jettiness subtractions for NNLO QCD calculations, JHEP 09 (2015) 058 [arXiv: 1505.04794] [INSPIRE].

[79] E.L. Berger, J. Gao, C.S. Li, Z.L. Liu and H.X. Zhu, Charm-quark production in deep-inelastic neutrino scattering at next-to-next-to-leading order in QCD, Phys. Rev. Lett. 116 (2016) 212002 [arXiv: 1601.05430] [INSPIRE].

[80] M. Cacciari, F.A. Dreyer, A. Karlberg, G.P. Salam and G. Zanderighi, Fully differential vector-boson-fusion Higgs production at next-to-next-to-leading order, Phys. Rev. Lett. 115 (2015) 082002 [Erratum ibid. 120 (2018) 139901] [arXiv: 1506.02660] [INSPIRE].

[81] J. Gao, C.S. Li and H.X. Zhu, Top quark decay at next-to-next-to leading order in QCD, Phys. Rev. Lett. 110 (2013) 042001 [arXiv:1210.2808] [INSPIRE].

[82] J. Butterworth et al., PDF4LHC recommendations for LHC run II, J. Phys. G 43 (2016) 023001 [arXiv: 1510.03865] [INSPIRE].

[83] J. Gao and P. Nadolsky, A meta-analysis of parton distribution functions, JHEP 07 (2014) 035 [arXiv: 1401.0013] [INSPIRE].

[84] L.A. Harland-Lang, A.D. Martin, P. Motylinski and R.S. Thorne, Parton distributions in the LHC era: MMHT 2014 PDFs, Eur. Phys. J. C 75 (2015) 204 [arXiv:1412.3989] [INSPIRE].

[85] NNPDF collaboration, Parton distributions for the LHC run II, JHEP 04 (2015) 040 [arXiv: 1410.8849] [INSPIRE].

[86] S. Dulat et al., New parton distribution functions from a global analysis of quantum chromodynamics, Phys. Rev. D 93 (2016) 033006 [arXiv:1506.07443] [INSPIRE].

[87] S. Carrazza, S. Forte, Z. Kassabov, J.I. Latorre and J. Rojo, An unbiased Hessian representation for Monte Carlo PDFs, Eur. Phys. J. C 75 (2015) 369 [arXiv:1505.06736] [INSPIRE].

[88] F. Maltoni, G. Ridolfi and M. Ubiali, b-initiated processes at the LHC: a reappraisal, JHEP 07 (2012) 022 [Erratum ibid. 04 (2013) 095] [arXiv:1203.6393] [INSPIRE]. 
[89] L.A. Harland-Lang, A.D. Martin, P. Motylinski and R.S. Thorne, Charm and beauty quark masses in the MMHT2014 global PDF analysis, Eur. Phys. J. C 76 (2016) 10 [arXiv: 1510.02332] [INSPIRE].

[90] E. Bothmann, F. Krauss and M. Schönherr, Single top-quark production with SHERPA, Eur. Phys. J. C 78 (2018) 220 [arXiv:1711.02568] [InSPIRE].

[91] J.M. Campbell, R.K. Ellis and C. Williams, Associated production of a Higgs boson at NNLO, JHEP 06 (2016) 179 [arXiv:1601.00658] [INSPIRE].

[92] R. Boughezal et al., Color singlet production at NNLO in MCFM, Eur. Phys. J. C 77 (2017) 7 [arXiv: 1605.08011] [INSPIRE].

[93] J. Alwall et al., The automated computation of tree-level and next-to-leading order differential cross sections, and their matching to parton shower simulations, JHEP $\mathbf{0 7}$ (2014) 079 [arXiv: 1405.0301] [INSPIRE].

[94] P. Artoisenet, R. Frederix, O. Mattelaer and R. Rietkerk, Automatic spin-entangled decays of heavy resonances in Monte Carlo simulations, JHEP 03 (2013) 015 [arXiv:1212.3460] [INSPIRE].

[95] T. Sjöstrand, S. Mrenna and P.Z. Skands, PYTHIA 6.4 physics and manual, JHEP 05 (2006) 026 [hep-ph/0603175] [INSPIRE].

[96] T. Sjöstrand et al., An introduction to PYTHIA 8.2, Comput. Phys. Commun. 191 (2015) 159 [arXiv: 1410.3012] [INSPIRE].

[97] M. Bahr et al., HERWIG++ physics and manual, Eur. Phys. J. C 58 (2008) 639 [arXiv: 0803.0883] [INSPIRE].

[98] E. Conte, B. Fuks and G. Serret, MadAnalysis 5, a user-friendly framework for collider phenomenology, Comput. Phys. Commun. 184 (2013) 222 [arXiv:1206.1599] [INSPIRE].

[99] M. Cacciari, G.P. Salam and G. Soyez, FastJet user manual, Eur. Phys. J. C 72 (2012) 1896 [arXiv: 1111.6097] [INSPIRE].

[100] T. Ježo, J.M. Lindert, P. Nason, C. Oleari and S. Pozzorini, An NLO+PS generator for $t \bar{t}$ and Wt production and decay including non-resonant and interference effects, Eur. Phys. J. C 76 (2016) 691 [arXiv:1607.04538] [INSPIRE].

[101] T. Liu, K. Melnikov and A.A. Penin, Nonfactorizable QCD effects in Higgs boson production via vector boson fusion, Phys. Rev. Lett. 123 (2019) 122002 [arXiv:1906.10899] [InSPIRE].

[102] F.A. Dreyer, A. Karlberg and L. Tancredi, On the impact of non-factorisable corrections in VBF single and double Higgs production, JHEP 10 (2020) 131 [arXiv:2005.11334] [INSPIRE]. 